\title{
ANALYTIC CYCLES IN FLIP PASSAGES AND IN INSTANTON MODULI SPACES OVER NON-KÄHLERIAN SURFACES
}

\author{
ANDREI TELEMAN
}

\begin{abstract}
Let $\mathcal{M}^{\text {st }}\left(\mathcal{M}^{\text {pst }}\right)$ be a moduli space of stable (polystable) bundles with fixed determinant on a complex surface with $b_{1}=1, p_{g}=0$, and let $Z \subset \mathcal{M}^{\text {st }}$ be a pure $k$-dimensional analytic set. We prove a general formula for the homological boundary $\delta[Z]^{B M} \in H_{2 k-1}^{B M}\left(\partial \hat{\mathcal{M}}^{\mathrm{pst}}, \mathbb{Z}\right)$ of the Borel-Moore fundamental class of $Z$ in the boundary of the blow up moduli space $\hat{\mathcal{M}}^{\text {pst }}$. The proof is based on the holomorphic model theorem of Te5, which identifies a neighborhood of a boundary component of $\hat{\mathcal{M}}^{\text {pst }}$ with a neighborhood of the boundary of a "blow up flip passage".

We then focus on a particular instanton moduli space which intervenes in our program for proving the existence of curves on class VII surfaces. Using our result, combined with general properties of the Donaldson cohomology classes, we prove incidence relations between the Zariski closures (in the considered moduli space) of certain families of extensions. These incidence relations are crucial for understanding the geometry of the moduli space, and cannot be obtained using classical complex geometric deformation theory.
\end{abstract}

\section{INTRODUCTION}

Let $(X, g)$ be a Gauduchon surface $\mathrm{Gau},(E, h)$ a Hermitian rank-2 bundle over $X, \mathcal{D}$ a holomorphic structure on the determinant line bundle $\operatorname{det}(E)$ and $a$ the Chern connection of the pair $(\mathcal{D}, \operatorname{det}(h))$. The moduli space $\mathcal{M}^{\text {pst }}\left(\mathcal{M}^{\text {st }}\right)$ of polystable (stable) holomorphic structures $\mathcal{E}$ on $E$ with $\operatorname{det}(\mathcal{E})=\mathcal{D}$ can be identified with the instanton moduli space $\mathcal{M}^{\mathrm{ASD}}$ (respectively $\mathcal{M}^{\mathrm{ASD}}{ }^{*}$ ) of (irreducible) projectively ASD unitary connections $A$ on $E$ with $\operatorname{det}(A)=a[\mathrm{DK},[\mathrm{Bu},[\mathrm{LT}]$, Te1], Te3], Te5]. The stable part $\mathcal{M}^{\text {st }} \subset \mathcal{M}^{\text {pst }}$ is open and has a natural complex space structure, which, in general, does not extend across the reduction locus $\mathcal{R}:=\mathcal{M}^{\text {pst }} \backslash \mathcal{M}^{\text {st }}$ (the subspace of reducible instantons).

The set of topological decompositions of $E$ (as direct sum of line bundles) can be identified with the set

$$
\operatorname{Dec}(E):=\left\{c \in H^{2}(X, \mathbb{Z}) \mid c\left(c_{1}(E)-c\right)=c_{2}(E)\right\} / \sim,
$$

where $\sim$ is the equivalence relation defined by the involution $c \mapsto c_{1}(E)-c$. We assume that $c_{1}(E) \notin 2 H^{2}(X, \mathbb{Z})$, which implies that this involution has no fixed points. Moreover, under this assumption, we showed [Te3], Te5] that, for a surface with $b_{1}(X)=1, p_{g}(X)=0$ (in particular for a class VII surface [BHPV], [Na]), $\mathcal{R}$ decomposes as a disjoint union of circles

$$
\mathcal{R}=\bigcup_{\lambda \in \mathcal{D e c}(E)} C_{\lambda}
$$

The author has been partially supported by the ANR project MNGNK, decision Nr. ANR-10BLAN-0118. 
where $C_{\lambda}:=\left\{[\mathcal{E}] \in \mathcal{M}^{\text {pst }} \mid \mathcal{E}\right.$ has a direct summand $\mathcal{L}$ with $\left.c_{1}(\mathcal{L}) \in \lambda\right\}$. Choosing a representative $c \in \lambda$, and putting $\mathfrak{d}:=\frac{1}{2} \operatorname{deg}_{g}(\mathcal{D}), C_{\lambda}$ can be identified with the circle

$$
C_{c}:=\left\{[\mathcal{L}] \in \operatorname{Pic}^{c}(X) \mid \operatorname{deg}_{g}(\mathcal{L})=\mathfrak{d}\right\}
$$

(see section 2.1). Blowing up $\mathcal{M}^{\text {pst }}$ at a circle $C_{\lambda}$ of regular reductions yields a proper map $p_{\lambda}: \hat{\mathcal{M}}_{\lambda}^{\text {pst }} \rightarrow \mathcal{M}^{\text {pst }}$, defined on a space $\hat{\mathcal{M}}_{\lambda}^{\text {pst }}$ which has a natural structure of a manifold with boundary around the exceptional locus $\mathcal{P}_{\lambda}:=p_{\lambda}^{-1}\left(C_{\lambda}\right)$ [Te5. This exceptional locus is a fiber bundle over $C_{\lambda}$ with a complex projective space as fiber. It's important to point out that, in our framework, a moduli space $\mathcal{M}^{\text {st }}$ contains distinguished locally closed complex subspaces $\mathcal{P}_{\varepsilon}^{c}$, which correspond to families of stable extensions, and are described in Remark 1.1 below. For $[\mathcal{L}] \in$ $\operatorname{Pic}(X)$ define

$$
\mathbb{P}_{[\mathcal{L}]}:=\left\{[\mathcal{E}] \in \mathcal{M}^{\text {st }} \mid \mathcal{E} \text { is an extension of } \mathcal{K} \otimes \mathcal{L}^{\vee} \text { by } \mathcal{L}\right\},
$$

and for a subset $A \subset \mathbb{R}$, and a class $c \in H^{2}(X, \mathbb{Z})$, put

$$
\operatorname{Pic}^{c}(X)_{A}:=\left\{[\mathcal{L}] \in \operatorname{Pic}^{c}(X) \mid \operatorname{deg}_{g}(\mathcal{L}) \in A\right\} .
$$

When $A$ is a singleton (an open interval), $\operatorname{Pic}^{c}(X)_{A}$ is a circle (an annulus).

Remark 1.1. Suppose that $b_{1}(X)=1, p_{g}(X)=0, c_{1}(E) \notin 2 H^{2}(X, \mathbb{Z})$, let $\lambda \in$ $\operatorname{Dec}(X)$ such that $C_{\lambda}$ is a circle of regular reductions, and choose $c \in \lambda$. Then

1. There exists an open neighborhood $\mathcal{U}_{\lambda}$ of $C_{\lambda}$ in $\mathcal{M}^{\text {pst }}$ such that $\mathcal{U}_{\lambda} \cap \mathcal{M}^{\text {st }}$ is a smooth complex manifold of dimension $4 c_{2}(E)-c_{1}^{2}(E)$,

2. There exists $\varepsilon>0$ such that

i) for any $[\mathcal{L}] \in \operatorname{Pic}^{c}(X)_{(\mathfrak{d}-\varepsilon, \mathfrak{d})}$ the subspace $\mathbb{P}_{[\mathcal{L}]}$ is a complex submanifold of $\mathcal{U}_{\lambda} \cap \mathcal{M}^{\text {st }}$. This submanifold can be identified with $\mathbb{P}\left(H^{1}\left(\mathcal{L}^{\otimes 2} \otimes \mathcal{K}^{\vee}\right)\right)$, where $\left.\operatorname{dim}\left(H^{1}\left(\mathcal{L}^{\otimes 2} \otimes \mathcal{K}^{\vee}\right)\right)\right)=r_{c}:=-\frac{1}{2}\left(2 c-c_{1}(E)\right)\left(2 c-c_{1}(E)+c_{1}(X)\right)$.

ii) The union $\bigcup_{[\mathcal{L}] \in \operatorname{Pic}^{c}(X)_{(\mathfrak{D}-\varepsilon, \mathfrak{o})}} \mathbb{P}_{[\mathcal{L}]}$ is disjoint, defines a smooth submanifold $\mathcal{P}_{\varepsilon}^{c}$ of $\mathcal{U}_{\lambda} \cap \mathcal{M}^{\text {st }}$, and the natural map $\mathcal{P}_{\varepsilon}^{c} \rightarrow \operatorname{Pic}^{c}(X)_{(\mathfrak{d}-\varepsilon, \mathfrak{d})}$ is a fiber bundle with fiber $\mathbb{P}_{\mathbb{C}}^{r_{c}-1}$.

The following remark highlights an advantage of the blow up moduli space:

Remark 1.2. With the notations, suppose $r_{c}>0$. For sufficiently small $\varepsilon>0$

1. The closure $\tilde{\mathcal{P}}_{\varepsilon}^{c}$ of $\mathcal{P}_{\varepsilon}^{c}$ in $\hat{\mathcal{M}}_{\lambda}^{\mathrm{pst}}$ is a $\mathbb{P}_{\mathbb{C}}^{r_{c}-1}$-bundle over $\operatorname{Pic}^{c}(X)_{[0-\varepsilon, 0]}$,

2. The closure $\overline{\mathcal{P}}_{\varepsilon}^{c}$ of $\mathcal{P}_{\varepsilon}^{c}$ in $\mathcal{M}^{\text {pst }}$ is obtained from $\tilde{\mathcal{P}}_{\varepsilon}^{c}$ be collapsing to points the fibers over the circle $\mathrm{Pic}_{\{\mathfrak{0}\}}^{c}$.

Note that, in general, is very hard to describe the Zariski closure of $\mathcal{P}_{\varepsilon}^{c}$ in $\mathcal{M}^{\text {st }}$, even when $X$ is a known surface (for instance a Kato surface). Understanding the Zariski closures of these families, and the incidence relations between these closures is very important for understanding the geometry of the moduli space, and plays an important role in our program to prove existence of curves on class VII surfaces. This is why we are interested in the general properties of the analytic subsets of $\mathcal{M}^{\text {st }}$. The main result of this article concerns the following

Problem 1. Let $Z \subset \mathcal{M}^{\text {st }}$ be a pure $k$-dimensional analytic set, and let $[Z]^{B M} \in$ $H_{2 k}^{B M}\left(\mathcal{M}^{\text {st }}, \mathbb{Z}\right)$ its fundamental class in Borel-Moore homology. Determine the homological boundary $\delta\left([Z]^{B M}\right) \in H_{2 k-1}\left(\mathcal{P}_{\lambda}, \mathbb{Z}\right)$ of $[Z]^{B M}$. 
Why is this problem relevant for understanding the geometry of an instanton moduli spaces $\mathcal{M}^{\text {pst }}$ and its families of extensions? In order to explain this, recall first $[\mathrm{DK}]$ that $\mathcal{M}^{\text {st }}=\mathcal{M}^{\mathrm{ASD}}{ }^{*}$ is naturally embedded in the infinite dimensional moduli space $\mathcal{B}^{*}$ of irreducible unitary connections $A$ on $E$ with $\operatorname{det}(A)=a$. The space $\mathcal{B}^{*}$ is endowed with tautological cohomology classes [DK], which will be called Donaldson classes. In general, a Donaldson class $\nu \in H^{*}\left(\mathcal{B}^{*}, \mathbb{Q}\right)$ cannot be extended across a circle of reductions, but it does extend to the exceptional locus $\mathcal{P}_{\lambda} \subset$ $\hat{\mathcal{M}}_{\lambda}^{\text {pst }}$ associated with a circle $C_{\lambda}$ of regular reductions. This a second important advantage of the blow up moduli space. With this remark we have

Proposition 4.1. Suppose that $b_{1}(X)=1, p_{g}(X)=0, c_{1}(E) \notin 2 H^{2}(X, \mathbb{Z}), \mathcal{M}^{\text {pst }}$ is compact, and all reductions in $\mathcal{M}^{\mathrm{pst}}$ are regular. For any Donaldson cohomology class $\nu \in H^{k-1}\left(\mathcal{B}^{*}, \mathbb{Q}\right)$ and any $\xi \in H_{k}^{B M}\left(\mathcal{M}^{\text {st }}, \mathbb{Q}\right)$ we have

$$
\sum_{\lambda \in \operatorname{Dec}(E)}\left\langle\nu_{\mathcal{P}_{\lambda}}, \delta_{\lambda} \xi\right\rangle=0
$$

where $\delta_{\lambda} \xi$ denotes the homological boundary of $\xi$ in $H_{k-1}\left(\mathcal{P}_{\lambda}, \mathbb{Q}\right)$.

The point is that the restrictions $\nu_{\mid \mathcal{P}_{\lambda}}$ have been computed explicitly [Te2, Corollary 2.6]. Therefore, assuming that Problem 1 is solved, formula (2) yields a strong obstruction to the existence of an analytic set $Z \subset \mathcal{M}^{\text {st }}$ with prescribed topological behavior around the circles of reductions $C_{\lambda}$. Note that (in the relevant cases) the particular moduli space $\mathcal{M}^{\text {pst }}$, used in our program to prove the existence of curves on class VII surfaces, satisfies the assumptions of Proposition $4.1 \mathrm{Te} 3$.

The main result of this article gives a solution to Problem 1. The proof is based on the holomorphic model theorem [Te5], which states that a neighborhood of the boundary $\mathcal{P}_{C}$ in $\mathcal{M}^{\text {pst }}$ can be identified with a neighborhood of the boundary of a standard model, which we called a blow up flip passage, and whose construction we recall briefly below.

Let $B$ be a Riemann surface, $p^{\prime}: E^{\prime} \rightarrow B, p^{\prime \prime}: E^{\prime \prime} \rightarrow B$ holomorphic Hermitian bundles of ranks $r^{\prime}, r^{\prime \prime}$, and $f: B \rightarrow \mathbb{R}$ a smooth function which is a submersion at any vanishing point, and such that $C:=f^{-1}(0)$ is a circle. The direct sum $E:=E^{\prime} \oplus E^{\prime \prime}$ is endowed with the $\mathbb{C}^{*}$-action $\left.\zeta \cdot\left(y^{\prime}, y^{\prime \prime}\right)\right)=\left(\zeta y^{\prime}, \zeta^{-1} y^{\prime \prime}\right)$. The zero locus $Z\left(m^{f}\right)$ of the map $m^{f}: E \rightarrow \mathbb{R}$ defined by

$$
m_{b}^{f}\left(y^{\prime}, y^{\prime \prime}\right)=\frac{1}{2}\left(\left\|y^{\prime}\right\|^{2}-\left\|y^{\prime \prime}\right\|^{2}\right)+f(b), \forall\left(y^{\prime}, y^{\prime \prime}\right) \in E_{b},
$$

is a smooth, $S^{1}$-invariant hypersurface. The induced $S^{1}$-action on $Z\left(m^{f}\right)$ is free away of $C$ (embedded in $E$ via the zero section). Put $F^{\prime}:=\left.E^{\prime}\right|_{C}, F^{\prime \prime}:=\left.E^{\prime \prime}\right|_{C}$. The normal bundle of $C$ in $Z\left(m^{f}\right)$ can be identified (as an $S^{1}$-bundle) with $F^{\prime} \oplus \bar{F}^{\prime \prime}$, hence the spherical blow up $\left.\widehat{Z\left(m^{f}\right)}\right)_{C}$ of $Z\left(m^{f}\right)$ at $C$ is a manifold with boundary, whose boundary can be identified with the sphere bundle $S\left(F^{\prime} \oplus \bar{F}^{\prime \prime}\right)$ (see section 2.1 in this article, Te5]). The blow up flip passage $\hat{Q}_{f}$ associated with the data $\left(p^{\prime}: E^{\prime} \rightarrow B^{\prime}, p^{\prime \prime}: E^{\prime \prime} \rightarrow B, f\right)$ is defined by

$$
\hat{Q}_{f}:=\widehat{Z\left(m^{f}\right)} C / S^{1} \text {. }
$$

This quotient is a smooth manifold whose boundary $\partial \hat{Q}_{f}$ can be identified with the projective bundle $\mathbb{P}\left(F^{\prime} \oplus \bar{F}^{\prime \prime}\right)$. The interior $\hat{Q}_{f} \backslash \partial \hat{Q}_{f}$ can be identified with the quotient $Q_{f}^{s}:=E_{f}^{s} / \mathbb{C}^{*}$, where $E_{f}^{s}:=\mathbb{C}^{*} \cdot\left(Z\left(m^{f}\right) \backslash C\right)$ is open in $E$. Therefore this 
interior comes with a natural complex structure. Note also that $\hat{Q}_{f}$ comes with a map $\mathfrak{q}: \hat{Q}_{f} \rightarrow B$, whose restriction to $Q_{f}^{s}$ is a holomorphic submersion. Choosing points $b_{ \pm} \in B_{ \pm}:=( \pm f)^{-1}(0, \infty)$ (and supposing $r^{\prime}>0, r^{\prime \prime}>0$ ), the fibers $\mathfrak{q}^{-1}\left(b_{ \pm}\right)$are smooth complex manifolds related by a flip. This explains the choice of the terminology "flip passage". More precisely, put $P^{\prime}:=\mathbb{P}\left(E^{\prime}\right), P^{\prime \prime}:=\mathbb{P}\left(E^{\prime \prime}\right)$ and let $P_{ \pm}^{\prime}\left(P_{ \pm}^{\prime \prime}\right)$ be the restriction of $P^{\prime}\left(P^{\prime \prime}\right)$ to $B_{ \pm}$. The projective bundles $P_{-}^{\prime}, P_{+}^{\prime \prime}$ are naturally embedded in $Q_{f}^{s}$. With these notations we see that the fiber $\mathfrak{q}^{-1}\left(b_{+}\right)$ is obtained from the fiber $\mathfrak{q}^{-1}\left(b_{-}\right)$by "replacing" $P_{b_{-}}^{\prime}$ with $P_{b_{+}}^{\prime \prime}$. We can state now

Problem 2. Let $Z \subset Q_{f}^{s}$ be a pure $k$-dimensional analytic set, and $[Z]^{B M} \in$ $H_{2 k}^{B M}\left(Q_{f}^{s}, \mathbb{Z}\right)$ its fundamental class in Borel-Moore homology. Determine the homological boundary $\delta\left([Z]^{B M}\right) \in H_{2 k-1}\left(\mathbb{P}\left(F^{\prime} \oplus \bar{F}^{\prime \prime}\right), \mathbb{Z}\right)$ of $[Z]^{B M}$.

The two problems Problem 1, Problem 2, are related by the the holomorphic model theorem proved in [Te5], which we explain briefly below (see section 2.2 for details). Coming back to our gauge theoretical framework, let $\lambda \in \operatorname{Dec}(E)$ with a circle of regular reductions $C_{\lambda}$, and fix $c \in \lambda$. The holomorphic model theorem gives a system $\left(p_{c}^{\prime}: E_{c}^{\prime} \rightarrow B_{c}, p_{c}^{\prime \prime}: E_{c}^{\prime \prime} \rightarrow B_{c}, f_{c}\right)$ as above, a diffeomorphism $\Psi_{c}$ between a neighborhood $O_{c}$ of $\partial \hat{Q}_{f_{c}}$ in $\hat{Q}_{f_{c}}$ and a neighborhood $\mathcal{O}_{\lambda}$ of $\mathcal{P}_{\lambda}$ in $\hat{\mathcal{M}}_{\lambda}^{\text {pst }}$, where $\hat{Q}_{f_{c}}$ is the blow up flip passage associated with $\left(p_{c}^{\prime}: E_{c}^{\prime} \rightarrow B_{c}, p_{c}^{\prime \prime}: E_{c}^{\prime \prime} \rightarrow\right.$ $\left.B_{c}, f_{c}\right)$. Moreover, $\Psi_{c}$ induces a diffeomorphism $\partial \hat{Q}_{f_{c}}=\mathbb{P}\left(F_{c}^{\prime} \oplus \bar{F}_{c}^{\prime \prime}\right) \stackrel{\simeq}{\longrightarrow} \mathcal{P}_{\lambda}$, a biholomorphism $O_{c} \backslash \partial \hat{Q}_{f_{c}} \stackrel{\simeq}{\rightarrow} \mathcal{O}_{\lambda} \backslash \mathcal{P}_{\lambda}$, and maps the projective bundles $P_{c,-}^{\prime}$, $P_{c,+}^{\prime \prime} \subset Q_{f_{c}}^{s}$ onto the extension spaces $\mathcal{P}_{\varepsilon}^{c}, \mathcal{P}_{\varepsilon}^{c_{1}(E)-c} \subset \mathcal{M}^{\text {st }}$ respectively.

We explain now our answer to Problem 2, which concerns an arbitrary blow up flip passage $\hat{Q}_{f}$ (see section 3.3). Let $\Theta^{\prime}, \Theta^{\prime \prime}$ be the tautological line bundles of the projective bundles $P^{\prime}:=\mathbb{P}\left(E^{\prime}\right), P^{\prime \prime}:=\mathbb{P}\left(E^{\prime \prime}\right)$, and denote by $Q$ the total space of the line bundle $p_{1}^{*}\left(\Theta^{\prime}\right) \otimes p_{2}^{*}\left(\Theta^{\prime \prime}\right)$ over the fiber product $P^{\prime} \times_{B} P^{\prime \prime}$. We identify this fiber product with the zero section of $Q$. One has a natural biholomorphism $j_{1}: Q \backslash\left(P^{\prime} \times_{B} P^{\prime \prime}\right) \rightarrow Q_{f}^{s} \backslash\left(P_{-}^{\prime} \cup P_{+}^{\prime \prime}\right)$.

Theorem 3.9. With the notations above, suppose $r^{\prime}>0, r^{\prime \prime}>0$, and let $Z \subset Q_{f}^{s}$ be an analytic subset of pure dimension $k \geq 1$ such that $\operatorname{dim}\left(Z \cap\left(P_{-}^{\prime} \cup P_{+}^{\prime \prime}\right)\right)<k$. Then

(1) The closure $\tilde{Z}$ of $j_{1}^{-1}\left(Z \backslash\left(P_{-}^{\prime} \cup P_{+}^{\prime \prime}\right)\right)$ in $Q$ is a pure $k$-dimensional analytic subset of $Q$ with $\operatorname{dim}\left(\tilde{Z} \cap\left(P^{\prime} \times_{B} P^{\prime \prime}\right)\right)<k$,

(2) Choosing a point $x \in C$, the equality

$$
\delta[Z]^{B M}=[C] \otimes J_{E_{x}^{\prime}}^{E_{x}^{\prime \prime}}\left([\tilde{Z}]^{B M} \cdot\left(P_{x}^{\prime} \times P_{x}^{\prime \prime}\right)\right)
$$

holds in $H_{1}(C, \mathbb{Z}) \otimes H_{2 k-2}\left(\mathbb{P}\left(F_{x}^{\prime} \oplus \bar{F}_{x}^{\prime \prime}\right), \mathbb{Z}\right)=H_{2 k-1}\left(\mathbb{P}\left(F^{\prime} \oplus \bar{F}^{\prime \prime}\right), \mathbb{Z}\right)$.

In this theorem $J_{E_{x}^{\prime \prime}}^{E_{x}^{\prime \prime}}: H_{2 s}\left(\mathbb{P}\left(E_{x}^{\prime}\right) \times \mathbb{P}\left(E_{x}^{\prime \prime}\right), \mathbb{Z}\right) \rightarrow H_{2 s+2}\left(\mathbb{P}\left(E_{x}^{\prime} \oplus \bar{E}_{x}^{\prime \prime}\right), \mathbb{Z}\right)$ denotes the join morphism defined in section 3.2 (see formula (17), Remark 3.7).

Our explicit applications concern the moduli space considered in our program for proving the existence of curves on class VII surfaces [Te3]. Let $(X, g)$ be a class VII surface endowed with a Gauduchon metric, $\mathcal{K}$ the canonical line bundle of $X, K$ its underlying differentiable line bundle, $(E, h)$ a Hermitian rank 2-bundle on $X$ with $c_{2}(E)=0$ and $\operatorname{det}(E)=K$, and $\mathcal{M}^{\text {st }}, \mathcal{M}^{\text {pst }}$ the two moduli spaces associated with the data $(X, g, E, h, \mathcal{K}) . \mathcal{M}^{\text {pst }}$ is always compact Te3, Theorem 
1.11]. Moreover, assuming that $\operatorname{deg}_{g}(\mathcal{K})<0, X$ is minimal and is not an Enoki surface, it follows that $\mathcal{M}^{\text {st }}$ is a smooth manifold of dimension $b_{2}(X)$, and all the reductions in $\mathcal{M}^{\mathrm{pst}}$ are regular Te3, Theorem 1.3]. We suppose for simplicity that $H_{1}(X, \mathbb{Z})=\mathbb{Z}$, which implies that $H^{2}(X, \mathbb{Z})$ is torsion free. Let $\left(e_{i}\right)_{1 \leq i \leq b_{2}(X)}$ be a Donaldson basis of $H^{2}(X, \mathbb{Z})$, i.e. a basis which satisfies the conditions

$$
e_{i} \cdot e_{j}=-\delta_{i j}, \sum_{i=1}^{b_{2}(X)} e_{i}=c_{1}(\mathcal{K}) .
$$

[Te3, section 1.1]. Put $\mathfrak{I}:=\left\{1, \ldots, b_{2}(X)\right\}$, and for $I \subset \mathfrak{I}$ put

$$
\bar{I}:=\mathfrak{I} \backslash I, e_{I}:=\sum_{i \in I} e_{i} .
$$

Since $c_{1}(E)=\sum_{i \in \mathfrak{I}} e_{i}$, we have $\mathcal{D} e c(E)=\left\{\left\{e_{I}, e_{\bar{I}}\right\} \mid I \subset \mathfrak{I}\right\}$, hence $\mathcal{M}^{\text {pst }}$ has $2^{b_{2}(X)-1}$ circles of reductions. Using the notation introduced in Remark 1.1, we have $r_{e_{I}}=|\bar{I}|$. Therefore, putting $\mathfrak{k}:=\frac{1}{2} \operatorname{deg}_{k}(\mathcal{K})$, we obtain, for every $I \subsetneq \mathfrak{I}$, a projective bundle $\mathcal{P}_{\varepsilon}^{e_{I}} \rightarrow \operatorname{Pic}^{e_{I}}(X)_{(\mathfrak{k}-\varepsilon, \mathfrak{k})}$ with fiber $\mathbb{P}_{\mathbb{C}}^{|\bar{I}|-1}$, and an embedding of $\mathcal{P}^{e_{I}}$ in $\mathcal{M}^{\text {st }}$. In particular $\mathcal{P}_{\varepsilon}^{0}$ is open in $\mathcal{M}^{\text {st }}$. This open subset defines an end (the "known end") of $\mathcal{M}^{\text {st }}$. As explained before, in the general case, for a given index set $I \subsetneq \mathfrak{I}$ (including for $\emptyset$ ) is very hard to describe the Zariski closure of $\mathcal{P}_{\varepsilon}^{e_{I}}$ in $\mathcal{M}^{\text {st }}$, even when $X$ is a known class VII surface. The result below, proved using the methods developed in this article, will be used in Te6.

Proposition 4.2, Let $X$ be a minimal class VII surface with $H_{1}(X, \mathbb{Z}) \simeq \mathbb{Z}$ and $b_{2}(X)=3$, which is not an Enoki surface. Let $g$ be a Gauduchon metric on $X$ with $\operatorname{deg}_{g}(\mathcal{K})<0$. Then

1. The component $\mathcal{M}_{0} \in \pi_{0}\left(\mathcal{M}^{\mathrm{pst}}\right)$ of $\mathcal{P}_{\varepsilon}^{0}$ contains all four circles of reductions,

2. Let $i \in \mathfrak{I}$. The Zariski closure of $\mathcal{P}_{\varepsilon}^{e_{i}}$ in $\mathcal{M}^{\text {st }}$ has pure dimension 2, does not intersect $\mathcal{P}_{\varepsilon}^{0}$, and contains the curve $\mathcal{P}_{\varepsilon}^{e_{I}}$ for a subset $I \subset \mathfrak{I}$ of cardinal 2.

If a stable bundle $\mathcal{E}$ is the central term of an extension of the form

$$
0 \rightarrow \mathcal{L} \rightarrow \mathcal{E} \rightarrow \mathcal{K} \otimes \mathcal{L}^{\vee} \rightarrow 0
$$

with $c_{1}(\mathcal{L})=e_{I}$, we agree to say that $\mathcal{E}$ is a stable extension of type $I$. The first statement of Proposition 4.2 shows that, for every $I \subsetneq \mathfrak{I}$ there exists a family of stable extensions of type $\emptyset$ which converges in $\mathcal{M}^{\text {st }}$ to a stable extension of type $I$. The second statement shows that for any $i \in \mathfrak{I}$ there exists $I_{i} \subset \mathfrak{I}$ of cardinal 2 , and a family of extensions of type $\{i\}$ which converges in $\mathcal{M}^{\text {st }}$ to a stable extension of type $I_{i}$. These statements cannot be proved using complex geometric arguments.

\section{FLIP PASSAGES AND THE HOLOMORPHIC MODEL THEOREM}

2.1. The spherical blow up. Let $M$ be an m-manifold, $W \subset M$ a closed $r$ dimensional submanifold. The spherical blow up $\hat{M}_{W}$ of $M$ at $W$ is a manifold with boundary, which comes with a smooth map $p: \hat{M}_{W} \rightarrow M$ with the following properties:

1. The boundary $\partial \hat{M}_{W}$ of $\hat{M}_{W}$ coincides with the sphere bundle $\left\{N_{W}^{M}\right\}^{*} / \mathbb{R}_{>0}$, where $\left\{N_{W}^{M}\right\}^{*}$ denotes the complement of the zero section in the normal bundle $N_{W}^{M}$ of $W$ in $M$, 
2. One has $p^{-1}(W)=\partial \hat{M}_{W}$, and the restriction $p_{\left.\right|_{\partial \hat{M}_{W}}: \partial \hat{M}_{W} \rightarrow W \text { coincides }}$ with the bundle projection $\left\{N_{W}^{M}\right\}^{*} / \mathbb{R}_{>0} \rightarrow W$,

3. The restriction $p_{\mid \hat{M}_{W} \backslash \partial \hat{M}_{W}}: \hat{M}_{W} \backslash \partial \hat{M}_{W} \rightarrow M \backslash W$ is a diffeomorphism.

We refer to $\mathrm{AK}$ for the construction and the functoriality properties of the spherical blow up. We will give now explicit constructions for the spherical blow up $\hat{M}_{W}$ in several situations which are of interest for us.

1. The case when $W=Z(s)$ for a regular section $s \in \Gamma(E)$.

Let $p: E \rightarrow M$ be a real rank $r$-bundle on $M$ and $s \in \Gamma(E)$ a section which is regular (transversal to the zero section) at any vanishing point. In this case the zero locus $W:=Z(s)$ is a smooth codimension $r$ submanifold of $M$, and the intrinsic derivative of $s$ defines an isomorphism $\left.N_{W}^{M} \rightarrow E\right|_{W}$. The spherical blow up $\hat{M}_{W}$ can be obtained as follows: endow $E$ with an Euclidean structure, and let $\pi: S(E) \rightarrow M$ be the corresponding sphere bundle. Then $\hat{M}_{W}$ can be identified with the submanifold

$$
\hat{s}:=\{(y, \rho) \in S(E) \times[0, \infty) \mid \rho y=s(\pi(y))\}
$$

of $S(E) \times[0, \infty)$. Note that the map $(y, \rho) \mapsto \rho y-s(\pi(y))$ is a section in the bundle $\left(\pi \circ p_{1}\right)^{*}(E)$ over $S(E) \times[0, \infty)$, and this section is transversal to the zero section of this pull back bundle. Via the identification $\hat{M}_{W}=\hat{s}$, the contraction map $\hat{s} \rightarrow M$ is the restriction $\left.\pi \circ p_{1}\right|_{\hat{s}}$.

2. The case when $M$ is the total space of a vector bundle $p: E \rightarrow B$, and $W=Z\left(\theta_{E}\right)$, where $\theta_{E} \in \Gamma\left(E, p^{*}(E)\right)$ is the tautological section of $E$.

The tautological section $\theta_{E} \in \Gamma\left(E, p^{*}(E)\right)$ of $E$ is defined by $\theta_{E}(y)=y$, where the right hand term is regarded as an element of the fiber $p^{*}(E)_{y}$. The zero locus of $\theta_{E}$ is the zero section $B \subset E$. This is a special case of 1 ., hence $\hat{E}_{B}=\hat{\theta}_{E}$. On the other hand $\hat{\theta}_{E}$ can be obviously identified with $S(E) \times[0, \infty)$ via the map $S(E) \times[0, \infty) \ni(y, \rho) \mapsto(\rho y, y, \rho) \in \hat{\theta}_{E}$. Therefore we get an identification

$$
\hat{E}_{B}=S(E) \times[0, \infty),
$$

and, via this identification, the contraction map $S(E) \times[0, \infty) \rightarrow E$ is $(y, \rho) \mapsto \rho y$.

Example 2.1. Consider the inclusion map $j: Z\left(m^{f}\right) \hookrightarrow E$ of the submanifold intervening in the definition of a blow up flip passage (see section 1, [Te5]). Then $j^{-1}(B)=C$ and $j$ induces a bundle isomorphism $N_{C}^{Z\left(m^{f}\right)}=\left.N_{B}^{E}\right|_{C}$. This implies that the blow up $\widehat{Z\left(m^{f}\right)_{C}}$ can be identified with the preimage of $Z\left(m^{f}\right)$ in the blow up $\hat{E}_{B}$ of $E$ at the zero locus of its tautological section. Since $\hat{E}_{B}=S(E) \times[0, \infty)$

$$
\widehat{Z\left(m^{f}\right)_{C}}=\left\{\left(\left(y^{\prime}, \bar{y}^{\prime \prime}\right), \rho\right) \in S\left(E^{\prime} \oplus \bar{E}^{\prime \prime}\right) \times[0, \infty) \mid m^{f}\left(\rho y^{\prime}, \rho y^{\prime \prime}\right)=0\right\} .
$$

3. The case when $M$ is the total space of a vector bundle $p: E \rightarrow B$, and $W=Z\left(\theta_{E}, p^{*}(s)\right)$, where $s \in \Gamma(F)$ is a section of a bundle $q: F \rightarrow B$.

Suppose $s \in \Gamma(F)$ is transversal to the zero section, and let $W:=Z(s)$ be its zero submanifold. Then $\left(\theta_{E}, p^{*}(s)\right) \in \Gamma\left(p^{*}(E) \oplus p^{*}(F)\right)$ is transversal to the zero section, $Z\left(\left(\theta_{E}, p^{*}(s)\right)\right.$ can be identified with $W$ via $p$, and one has an obvious identification 
$N_{W}^{E}=\left.(E \oplus F)\right|_{W}$. In the same way as in 2. we get an identification

$$
\hat{E}_{W}=\{((y, v), \rho) \in S(E \oplus F) \times[0, \infty) \mid \rho v=s(p(y))\},
$$

and, via this identification, the contraction map $\hat{E}_{W} \rightarrow E$ is $((y, v), \rho) \mapsto \rho y$.

2.2. The holomorphic model theorem. Let $X$ be a surface with $b_{1}(X)=1$, $p_{g}=0$. For such a surface the canonical morphism $H^{1}(X, \mathbb{C}) \rightarrow H^{1}\left(X, \mathcal{O}_{X}\right)$ is an isomorphism, hence $\operatorname{Pic}^{0}(X)=H^{1}\left(X, \mathcal{O}_{X}\right) / H^{1}(X, \mathbb{Z}) \simeq \mathbb{C}^{*}$.

Fix $x_{0} \in X$, and let $\mathscr{L}$ be the Poincaré line bundle normalized at $x_{0}$ on the product $\operatorname{Pic}(X) \times X$. Let $p_{1}: \operatorname{Pic}(X) \times X \rightarrow \operatorname{Pic}(X), p_{2}: \operatorname{Pic}(X) \times X \rightarrow X$ be the two projections. The $H^{1}(\operatorname{Pic}(X), \mathbb{Z}) \otimes H^{1}(X, \mathbb{Z})$-term of the Künneth decomposition of $c_{1}(\mathscr{L})$ can be interpreted as a morphism $\delta: H_{1}(X, \mathbb{Z}) \rightarrow H^{1}(\operatorname{Pic}(X), \mathbb{Z})$.

Fix a Gauduchon metric $g$ on $X$. For any $c \in H^{2}(X, \mathbb{Z}), t \in \mathbb{R}$ the level set $\operatorname{Pic}^{c}(X)_{\{t\}}:=\left\{\left.\operatorname{deg}_{g}\right|_{\operatorname{Pic}^{c}(X)}\right\}^{-1}(t)$ is a circle, and $\delta$ induces an isomorphism $H_{1}(X, \mathbb{Z}) \rightarrow H^{1}\left(\operatorname{Pic}^{c}(X)_{\{t\}}, \mathbb{Z}\right)$. We will always endow $\operatorname{Pic}^{c}(X)_{\{t\}}$ with the boundary orientation of $\partial \operatorname{Pic}^{c}(X)_{[t,+\infty)}$. With this convention we get an isomorphism $H_{1}(X, \mathbb{Z}) \rightarrow \mathbb{Z}$ given by $h \mapsto\left\langle\delta(h),\left[\operatorname{Pic}^{c}(X)_{\{t\}}\right]\right\rangle$, which is independent of $c \in$ $H^{2}(X, \mathbb{Z}), t \in \mathbb{R}$ and the Gauduchon metric $g$. This isomorphism defines a distinguished generator $\gamma_{X} \in H^{1}(X, \mathbb{Z})$. Therefore, by definition, we have

$$
\left\langle\gamma_{X}, h\right\rangle=\left\langle\delta(h),\left[\operatorname{Pic}^{c}(X)_{\{t\}}\right]\right\rangle \forall h \in H_{1}(X, \mathbb{Z}) .
$$

Let $E$ be a Hermitian rank 2-bundle with $c_{1}(E) \notin 2 H^{2}(X, \mathbb{Z})$ on $X, \mathcal{D}$ a holomorphic structure on $\operatorname{det}(E), \lambda \in \operatorname{Dec}(E)$, and $c \in \lambda$. We define the harmonic map $f_{c}: \operatorname{Pic}^{c}(X) \rightarrow \mathbb{R}$ by $f_{c}([\mathcal{L}]):=\pi\left(\operatorname{deg}_{g}(\mathcal{L})-\mathfrak{d}\right)$, where $\mathfrak{d}:=\frac{1}{2} \operatorname{deg}_{g}(\mathcal{D})$. The vanishing circle $C_{c}:=f_{c}^{-1}(0)=\operatorname{Pic}^{c}(X)_{\{\mathfrak{0}\}}$ can be identified with the reduction circle $C_{\lambda} \subset \mathcal{M}_{\mathcal{D}}^{\text {pst }}(E)$ via the isomorphism $k_{c}: C_{c} \rightarrow C_{\lambda}$ given by $k_{c}([\mathcal{L}]):=\left[\mathcal{L} \oplus\left(\mathcal{D} \otimes \mathcal{L}^{\vee}\right)\right]$. Using the Riemann-Roch and Grauert's theorems we obtain [Te5, Proposition 3.3]:

Remark 2.1. Suppose $C_{\lambda}$ is a circle of regular reductions. There exists a Zariski open neighborhood $U$ of $C_{c}$ in $\operatorname{Pic}^{c}(X)$ such that the restrictions of the coherent sheaves $R^{1} p_{1 *}\left(\mathscr{L}^{\otimes 2} \otimes p_{2}^{*}\left(\mathcal{D}^{\vee}\right)\right), R^{1} p_{1 *}\left(\mathscr{L}^{\otimes-2} \otimes p_{2}^{*}(\mathcal{D})\right)$ to $U$ are locally free of ranks

$r_{c}^{\prime}=-\frac{1}{2}\left(2 c-c_{1}(E)\right)\left(2 c-c_{1}(E)+c_{1}(X)\right), r_{c}^{\prime \prime}=-\frac{1}{2}\left(-2 c+c_{1}(E)\right)\left(-2 c+c_{1}(E)+c_{1}(X)\right)$ respectively, and the fibers of the corresponding bundles at a point $y \in U$ are identified with $H^{1}\left(\mathscr{L}_{y}^{\otimes 2} \otimes p_{2}^{*}\left(\mathcal{D}^{\vee}\right)\right), H^{1}\left(\mathscr{L}_{y}^{\otimes-2} \otimes p_{2}^{*}(\mathcal{D})\right)$.

Therefore, for sufficiently small $\varepsilon>0$, we obtain holomorphic bundles $E_{c}^{\prime}, E_{c}^{\prime \prime}$ of ranks $r_{c}^{\prime}, r_{c}^{\prime \prime}$ on the annulus $B_{c}:=\operatorname{Pic}^{c}(X)_{(\mathfrak{d}-\varepsilon, \mathfrak{d}+\varepsilon)}=f_{c}^{-1}(-\pi \varepsilon, \pi \varepsilon)$.

The restrictions $\left.E_{c}^{\prime}\right|_{C_{c}},\left.E_{c}^{\prime \prime}\right|_{C_{c}}$ can be endowed with natural Hermitian metrics, by identifying them with suitable harmonic spaces [Te5]. Using Te5, Remark $2.8]$ we obtain a well defined blow up flip passage, which will be denoted by $\hat{Q}_{f_{c}}$. Correspondingly, we put $F_{c}^{\prime}:=\left.E_{c}^{\prime}\right|_{C_{c}}, F_{c}^{\prime \prime}:=\left.E_{c}^{\prime \prime}\right|_{C_{c}}, P_{c}^{\prime}:=\mathbb{P}\left(E_{c}^{\prime}\right), P_{c}^{\prime \prime}:=\mathbb{P}\left(E_{c}^{\prime \prime}\right)$, $P_{c, \pm}^{\prime}:=\left.P_{c}^{\prime}\right|_{B_{c, \pm}}, P_{c, \pm}^{\prime \prime}:=\left.P_{c}^{\prime \prime}\right|_{B_{c, \pm}}$.

Theorem 2.2. Under the assumptions and with the notations above there exists an open neighborhood $O_{c}$ of $\partial \hat{Q}$ in $\hat{Q}_{f_{c}}$ and a diffeomorphism $\Psi_{c}: O_{c} \rightarrow \mathcal{O}_{\lambda}$ onto a smooth open neighborhood $\mathcal{O}_{\lambda}$ of $\mathcal{P}_{\lambda}$ in $\hat{\mathcal{M}}_{\lambda}^{\text {pst }}$ such that 
1. $\Psi_{c}$ induces a smooth bundle isomorphism $\partial \Psi_{c}$ which fits in the commutative diagram

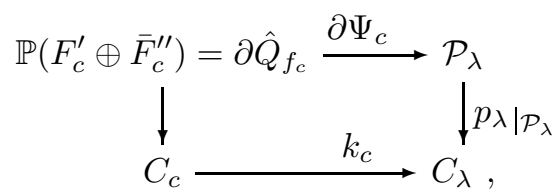

2. $\Psi_{c}$ induces a biholomorphism $O_{c} \backslash \partial \hat{Q}_{f_{c}} \rightarrow \mathcal{O}_{\lambda} \backslash \mathcal{P}_{\lambda}$,

3. $\Psi_{c}\left(P_{c-}^{\prime}\right)=\mathcal{P}_{\varepsilon}^{c} \cap \mathcal{O}_{\lambda}, \Psi_{c}\left(P_{c+}^{\prime \prime}\right)=\mathcal{P}_{\varepsilon}^{c_{1}(E)-c} \cap \mathcal{O}_{\lambda}$,

4. Denoting by $\nu(h), \nu(u)$ the Donaldson classes associated with $h \in H_{1}(X, \mathbb{Q})$, $u \in H_{2}(X, \mathbb{Q})$, we have

$$
\left(\partial \Psi_{c}\right)^{*}(\nu(h))=\delta(h) \otimes W_{c},\left(\partial \Psi_{c}\right)^{*}(\nu(u))=\frac{1}{2}\left\langle 2 c-c_{1}(E), u\right\rangle W_{c},
$$

where $W_{c}$ is the positive generator of $H_{2}\left(\mathbb{P}\left(F_{c}^{\prime} \oplus \bar{F}_{c}^{\prime \prime}\right), \mathbb{Z}\right)$.

The first two statements are proved in [Te5], and the third can be proved easily using the construction of $\Psi_{c}$. The fourth follows from [Te2, Corollary 2.6].

\section{The homological boundary of an analytic set $Z \subset Q_{f}^{\text {st }}$}

3.1. The real blow up of a Borel-Moore homology class and its boundary. We start with a brief account on the well known intersection theory in Borel-Moore theory (see for instance $\mathrm{BH}$, section 1.12]) in a very particular framework. Since in our results the signs play a crucial role, and in the literature one can find different conventions for the relevant objects intervening in our formulae (the cap product, the orientation of the normal bundle of an oriented submanifold of an oriented manifold, the Thom isomorphism), we will write down carefully the formulae we need.

In general, if $M$ is a differentiable manifold, and $Y \subset M$ a submanifold of $M$, we will always use the direct sum decomposition $\left.T_{M}\right|_{Y}=T_{Y} \oplus N_{Y}^{M}$ (defined by a Riemannian metric on $M$ ) to orient anyone of the three objects $M, Y, N_{Y}^{M}$ using orientations of the other two. In particular, the total space $E$ of an oriented Euclidean bundle over an oriented base $Y$ is oriented such that the obvious isomorphism $\left.T_{E}\right|_{Y}=T_{Y} \oplus E$ is orientation preserving. Note that $\left.\mathrm{BT}\right]$ uses a different convention.

For an oriented $r$-dimensional Euclidean vector space $F$ we denote by $B(F)$ the open unit ball of $F$, by $[F, F \backslash B(F)]$ the generator of $H_{r}(F, F \backslash B(F), \mathbb{Z})$ defined by the fixed orientation, and by $\{F, F \backslash B(F)\}$ the corresponding generator of $H^{r}(F, F \backslash B(F), \mathbb{Z})$.

Let $p: E \rightarrow Y$ be an oriented Euclidean rank $r$-bundle over a connected, oriented, closed manifold $Y$. We denote by $\Phi_{E}$ the section in the local coefficient system

$$
x \mapsto H_{r}\left(E_{x}, E_{x} \backslash B\left(F_{x}\right), \mathbb{Z}\right)
$$

given by $x \mapsto\left[E_{x}, E_{x} \backslash B\left(F_{x}\right)\right]$, by $B(E)$ the unit ball bundle of $E$, and by $\phi_{E} \in$ $H^{r}(E, E \backslash B(E), \mathbb{Z})$ its Thom class. For any $k \in \mathbb{N}$ the morphism

$$
\tau_{k}^{E}: H_{r+k}(E, E \backslash B(E), \mathbb{Z}) \rightarrow H_{k}(Y, \mathbb{Z}), \tau_{k}^{E}(u):=(-1)^{k r} p_{*}\left(\phi_{E} \cap u\right)
$$

is an isomorphism [Sp, Theorem 5.7.10]. In this formula, on the right, we used the sign convention of [Do, Section VII.12] for the definition of the cap product, which 
does not agree with the conventions used in $\left[\mathrm{Sp},[\mathrm{BH}]\right.$. The factor $(-1)^{k r}$ has been inserted in order to recover the standard isomorphism

$$
h \otimes[F, F \backslash B(F)] \mapsto h
$$

in the case of the trivial bundle $E=Y \times F \stackrel{p_{1}}{\longrightarrow} Y$. Having this case in mind, put

$$
h \otimes \Phi_{E}:=\left\{\tau_{k}^{E}\right\}^{-1}(h) .
$$

We will identify $H_{*}(F, F \backslash B(F), \mathbb{Z}), H_{*}(E, E \backslash B(F), \mathbb{Z})$ with the Borel-Moore homology $H_{*}^{B M}(B(F), \mathbb{Z}), H_{*}^{B M}(B(E), \mathbb{Z})$ of $F$ and $E$ respectively via the canonical isomorphisms

$$
\begin{gathered}
H_{*}(F, F \backslash B(F), \mathbb{Z}) \rightarrow H_{*}(\bar{B}(F), S(F), \mathbb{Z}) \rightarrow H_{*}^{B M}(B(F), \mathbb{Z}), \\
H_{*}(E, E \backslash B(E), \mathbb{Z}) \rightarrow H_{*}(\bar{B}(E), S(E), \mathbb{Z}) \rightarrow H_{*}^{B M}(B(E), \mathbb{Z})
\end{gathered}
$$

$\mathrm{BH}$, Section 1.2]. Via the first identification, $[F, F \backslash B(F)]$ corresponds to the fundamental class $[B(F)]$ of the oriented manifold $F$ in Borel-Moore homology, hence $\Phi_{E}$ corresponds to the section $\mathfrak{B}_{E}$ of the local coefficient system

$$
x \mapsto H_{r}^{B M}\left(B\left(E_{x}\right), \mathbb{Z}\right)
$$

given by $x \mapsto\left[B\left(E_{x}\right)\right]$. Therefore the isomorphism $\tau_{k}^{E}$ induces an isomorphism $\tau_{k}^{B(E)}: H_{r+k}^{B M}(E, \mathbb{Z}) \rightarrow H_{k}(Y, \mathbb{Z})$, and we can put

$$
h \otimes \mathfrak{B}_{E}:=\left\{\tau_{k}^{B(E)}\right\}^{-1}(h) .
$$

By our orientation convention for $E$ (and implicitly $B(E)$ ), the fundamental class of $B(E)$ in Borel-Moore homology will be given by $[B(E)]=[Y] \otimes \mathfrak{B}_{E}$. With these conventions, the morphism $p^{*}: H^{*}(Y, \mathbb{Z}) \rightarrow H^{*}(B(E), \mathbb{Z})$ commutes with the Poincaré duality isomorphisms on $Y$ and $B(E)$, i.e. one has the identity

$$
p^{*}(b) \cap[B(E)]=(b \cap[Y]) \otimes \mathfrak{B}_{E} \forall b \in H^{*}(Y, \mathbb{Z}) .
$$

Definition 3.1. Let $M$ be a connected, oriented m-dimensional manifold. For a class $c \in H_{k}^{B M}(M, \mathbb{Z})$ and a closed, oriented l-dimensional submanifold $W \subset M$ we define the homological intersection of $c$ with $W$ by

$$
c \cdot W:=j_{W}^{*}(b) \cap[W] \in H_{l+k-m}(W, \mathbb{Z}),
$$

where $j_{W}: W \hookrightarrow M$ is the inclusion map, and $b \in H^{m-k}(M, \mathbb{Z})$ is the Poincaré dual of $c$, i.e. one has $c=b \cap[M]$.

Remark 3.2. In the conditions of Definition 3.1 it holds

1. If $c$ is the fundamental class of a closed, oriented submanifold $Z \subset M$ which intersects $W$ transversally, then one has

$$
c \cdot W=(-1)^{(m-l)(m-k)}[W \cap Z],
$$

where $T=W \cap Z$ is regarded as a submanifold of $W$ endowed with the orientation induced by the orientation of $W$, and the orientation of $N_{W \cap Z}^{W}$ given by the natural isomorphism $N_{W \cap Z}^{W}=\left.N_{Z}^{M}\right|_{W \cap Z}$.

2. If $M$ is a complex manifold, $W$ a complex submanifold, and $c$ the fundamental class of an $k$-dimensional analytic subset $Z \subset M$ such that $W \cap Z$ has pure dimension $l+k-m$, then $c \cdot W$ is the fundamental class of the analytic cycle $W \cap Z$ of $W$. 
The unit sphere $S(F)$ of an oriented Euclidean space $F$ is given the boundary orientation of the closed unit disk $\bar{B}(F)$, i.e. the orientation for which the obvious isomorphism $\left.\mathbb{R} \eta \oplus T_{S(F)} \simeq T_{F}\right|_{S(F)}$ is orientation preserving, where $\eta$ is the outer normal field of $S(F)$. The sphere bundle $S(E)$ of an oriented Euclidean bundle $p: E \rightarrow Y$ is oriented using the same rule fiberwise. We denote by $\mathfrak{S}_{E}$ the section of the local coefficient system $x \mapsto H_{r-1}\left(S\left(E_{x}\right), \mathbb{Z}\right)$ given by $x \mapsto\left[S\left(E_{x}\right)\right]$. Using the Leray-Hirsch theorem again, and putting $n:=\operatorname{dim}(Y)$, we get as above an isomorphism

$$
H_{n+r-1}(S(E)) \simeq H_{n}(Y) \otimes H_{r-1}\left(S^{r-1}, \mathbb{Z}\right)
$$

and an identification $[S(E)]=[Y] \otimes \mathfrak{S}_{E}$. Using the Borel-Moore long exact sequence

$$
\cdots \rightarrow H_{j}^{B M}(S(E), \mathbb{Z}) \rightarrow H_{j}^{B M}(\bar{B}(E), \mathbb{Z}) \rightarrow H_{j}^{B M}(B(E), \mathbb{Z}) \stackrel{\partial}{\longrightarrow} H_{j-1}^{B M}(S(E), \mathbb{Z})
$$

associated with the open embedding $B(E) \hookrightarrow \bar{B}(E)[\mathrm{BH}$, Section 1.6], and comparing it with the long exact sequence of the pair $(\bar{B}(E), S(E))$, we get

$$
\partial\left(h \otimes \mathfrak{B}_{E}\right)=(-1)^{s} h \otimes \mathfrak{S}_{E} \forall h \in H_{s}(Y, \mathbb{Z}) .
$$

In particular $\partial[B(E)]=\partial\left([Y] \otimes \mathfrak{B}_{E}\right)=(-1)^{n}[Y] \otimes \mathfrak{S}_{E}=(-1)^{n}[S(E)]$.

Let $M$ be an oriented Riemannian $m$-dimensional manifold and $W \subset M$ a closed, oriented $l$-dimensional submanifold. Let $\hat{M}_{W}$ be the spherical blow up of $M$ with center $W$. If we omit orientations, the boundary $\partial M_{W}$ can be identified with the sphere bundle $S\left(N_{W}\right)$ of the normal bundle $N_{W}$ of $W$ in $M$.

Let $c \in H_{k}^{B M}(M, \mathbb{Z})$ be a $k$-dimensional Borel-Moore homology class. The image $c^{W}:=c_{M \backslash W}$ of $c$ under the canonical morphism $H_{k}^{B M}(M, \mathbb{Z}) \rightarrow H_{k}^{B M}(M \backslash W, \mathbb{Z})$ can be regarded as a $k$-dimensional Borel-Moore homology class of the interior $\hat{M}_{W} \backslash \partial \hat{M}_{W}$ of the manifold with boundary $\hat{M}_{W}$. Our problem is to compute explicitly the boundary $\delta\left(c^{W}\right) \in H_{k-1}^{B M}\left(\partial \hat{M}_{W}, \mathbb{Z}\right)$ in terms of topological invariants of the triple $(M, W, c)$.

Let $K$ be a compact tubular neighborhood of $W$, denote by $U$ its interior and by $c_{U}, c_{M \backslash K}$ the images of $c$ in $H_{k}^{B M}(U, \mathbb{Z}), H_{k}^{B M}(M \backslash K, \mathbb{Z})$ via the canonical morphisms. The Borel-Moore long exact sequence associated with the open embedding $U \cup(M \backslash K) \hookrightarrow M$ contains the segment

$$
\cdots \rightarrow H_{k}^{B M}(M, \mathbb{Z}) \rightarrow H_{k}^{B M}(U \cup(M \backslash K), \mathbb{Z}) \stackrel{\delta}{\longrightarrow} H_{k-1}(\partial K, \mathbb{Z}) \rightarrow \ldots,
$$

which shows that

$$
\delta\left(c_{U}\right)+\delta\left(c_{M \backslash K}\right)=0 .
$$

Using the obvious identification $H_{*}^{B M}\left(\partial \hat{M}_{W}, \mathbb{Z}\right)=H_{*}^{B M}(\partial K, \mathbb{Z})$, we obtain

$$
\delta\left(c^{W}\right)=-\delta\left(c_{U}\right) \text {. }
$$

Write $c=b \cap[M]$, where $b \in H^{m-k}(M, \mathbb{Z})$ is the inverse image of $v$ via the Poincaré duality isomorphism. Denoting by $j_{U}: U \hookrightarrow M, j_{W}: W \hookrightarrow M$ the embedding maps, by $p_{W}^{U}: U \rightarrow W$ the projection map, and using formula (7), we get

$c_{U}=j_{U}^{*}(b) \cap[U]=\left(p_{W}^{U}\right)^{*}\left(j_{W}^{*}(b)\right) \cap[U]=\left(j_{W}^{*}(b) \cap[W]\right) \otimes \mathfrak{B}_{N_{W}}=(c \cdot W) \otimes \mathfrak{B}_{N_{W}}$. Therefore, by (8) we get $\delta\left(c_{U}\right)=(-1)^{l+k-m}(c \cdot W) \otimes \mathfrak{S}_{N_{W}}$. This proves

Proposition 3.3. Under the assumptions and with the notations above one has

$$
\delta\left(c^{W}\right)=(-1)^{l+k-m+1}(c \cdot W) \otimes \mathfrak{S}_{N_{W}} .
$$


Example 3.1. For the special case $k=m, c=[M]^{B M}$ we have $b=1 \in H^{0}(M)$ and $c \cdot W=[W]$, hence

$$
\delta\left([M]^{W}\right)=(-1)^{l+1}[W] \otimes \mathfrak{S}_{N_{W}} .
$$

3.2. The join morphisms $H_{k}(\mathbb{P}(U) \times \mathbb{P}(V), \mathbb{Z}) \rightarrow H_{k+2}(\mathbb{P}(U \oplus V), \mathbb{Z})$. Let $G$ be a Lie group and $F$ a differentiable manifold.

Definition 3.4. The F-bundle associated with a left principal $G$-bundle $P \rightarrow B$ and a left action $\alpha: G \times F \rightarrow F$ is defined by $P \times{ }_{\alpha} V:=P \times V / G$ where $P \times V$ is endowed with the diagonal left $G$-action.

The standard example is the tautological line bundle $\Theta_{U}$ on the projective space $\mathbb{P}(U)$ of a finite dimensional complex vector space $U$. Denoting by $\chi_{0}$ the standard character $\mathbb{C}^{*} \rightarrow \mathbb{C}^{*}=\mathrm{GL}(1, \mathbb{C})$ given by $\zeta \mapsto \zeta$, one has

$$
\Theta_{U}=U^{*} \times{ }_{\chi_{0}^{-1}} \mathbb{C}
$$

Let $U, V$ be finite dimensional complex vector spaces. For a subset $A \subset \mathbb{P}(U) \times \mathbb{P}(V)$ we define its set theoretical join $J_{U V}(A) \subset \mathbb{P}(U \oplus V)$ by

$$
J_{U V}(A):=\bigcup_{([u],[v]) \in A} \mathbb{P}(\mathbb{C} u \oplus \mathbb{C} v) .
$$

We chose the word "join" because $\mathbb{P}(\mathbb{C} u \oplus \mathbb{C} v)$ is the line joining the pair of points $[u, 0],[0, v] \in \mathbb{P}(U \oplus V)$, hence $J_{U, V}(A)$ is the union of joining lines associated with pairs $([u],[v]) \in A$. Consider the projective line bundle

$$
p_{U V}: \mathbb{P}\left(p_{1}^{*}\left(\Theta_{U}\right) \oplus p_{2}^{*}\left(\Theta_{V}\right)\right) \rightarrow \mathbb{P}(U) \times \mathbb{P}(V)
$$

on $\mathbb{P}(U) \times \mathbb{P}(V)$. Using Definition 3.4 the total space $\mathbb{P}\left(p_{1}^{*}\left(\Theta_{U}\right) \oplus p_{2}^{*}\left(\Theta_{V}\right)\right)$ can be identified with the associated bundle $\left(U^{*} \times V^{*}\right) \times{ }_{\alpha} \mathbb{P}^{1}$, where $\alpha:\left(\mathbb{C}^{*} \times \mathbb{C}^{*}\right) \times \mathbb{P}^{1} \rightarrow \mathbb{P}^{1}$ acts by

$$
\alpha\left(\left(\zeta_{1}, \zeta_{2}\right),\left[z_{0}, z_{1}\right]\right)=\left[\zeta_{1}^{-1} z_{0}, \zeta_{1}^{-1} z_{1}\right] .
$$

The map $q_{U V}: \mathbb{P}\left(p_{1}^{*}\left(\Theta_{U}\right) \oplus p_{2}^{*}\left(\Theta_{V}\right)\right) \rightarrow \mathbb{P}(U \oplus V)$ given by

$$
q_{U V}\left(\left[(u, v),\left[z_{0}, z_{1}\right]\right]\right):=\left[z_{0} u, z_{1} v\right] \quad \forall(u, v) \in U^{*} \times V^{*}, \forall\left[z_{0}, z_{1}\right] \in \mathbb{P}_{\mathbb{C}}^{1}
$$

is a modification which blows up the linear subvarieties $\mathbb{P}(U \times\{0\}), \mathbb{P}(\{0\} \times V)$ of $\mathbb{P}(U \oplus V)$. We obtain the diagram

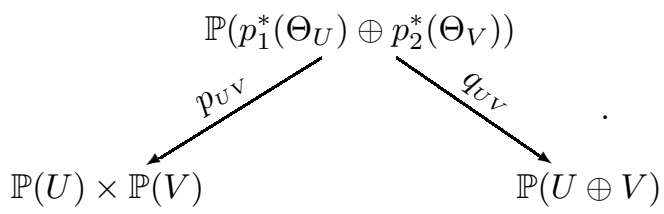

With these notations one has

$$
J_{U V}(A)=q_{U V}\left(p_{U V}^{-1}(A)\right) .
$$

This formula allows us to define a homological version of the join map. The Gysin exact sequence $[\mathrm{Sp}$, Theorem 9.3.3] of the sphere bundle

$$
p_{U V}: \mathbb{P}\left(p_{1}^{*}\left(\Theta_{U}\right) \oplus p_{2}^{*}\left(\Theta_{V}\right)\right) \rightarrow \mathbb{P}(U) \times \mathbb{P}(V)
$$

contains the segment

$$
H_{2 k+3}(\mathbb{P}(U) \times \mathbb{P}(V), \mathbb{Z}) \stackrel{\partial}{\rightarrow} H_{2 k}(\mathbb{P}(U) \times \mathbb{P}(V), \mathbb{Z}) \stackrel{\sigma_{U V}}{\longrightarrow} H_{2 k+2}\left(\mathbb{P}\left(p_{1}^{*}\left(\Theta_{U}\right) \oplus p_{2}^{*}\left(\Theta_{V}\right)\right), \mathbb{Z}\right)
$$




$$
\stackrel{p_{U V *}}{\longrightarrow} H_{2 k+2}(\mathbb{P}(U) \times \mathbb{P}(V), \mathbb{Z}) \stackrel{\partial}{\longrightarrow} H_{2 k-1}(\mathbb{P}(U) \times \mathbb{P}(V), \mathbb{Z}) \rightarrow \ldots
$$

Since the odd dimensional homology of $\mathbb{P}(U) \times \mathbb{P}(V)$ vanishes, it follows that $\sigma_{U V}$ is a monomorphism, more precisely identifies $H_{2 k}(\mathbb{P}(U) \times \mathbb{P}(V), \mathbb{Z})$ with

$$
\operatorname{ker}\left(p_{U V *}: H_{2 k+2}\left(\mathbb{P}\left(p_{1}^{*}\left(\Theta_{U}\right) \oplus p_{2}^{*}\left(\Theta_{V}\right)\right), \mathbb{Z}\right) \rightarrow H_{2 k+2}(\mathbb{P}(U) \times \mathbb{P}(V), \mathbb{Z})\right) .
$$

This morphism is defined using the homology spectral sequence associated with the the sphere bundle $p_{U V}$ in the following way (see the proof of $\mathrm{Sp}$, Theorem 9.3.3]). The local coefficient system

$$
\mathbb{P}(U) \times \mathbb{P}(V) \ni([u],[v]) \rightarrow H^{2}(\mathbb{P}(\mathbb{C} u \oplus \mathbb{C} v))
$$

comes with a natural section $\mathfrak{S}$ defined by the fundamental classes of the complex lines $\mathbb{P}(\mathbb{C} u \oplus \mathbb{C} v)$, hence this local coefficient system can be written formally as $\mathbb{Z} \mathfrak{S}$. The morphism $\sigma_{U V}$ is induced by the composition

$$
E_{2 k, 2}^{2}=H_{2 k}(\mathbb{P}(U) \times \mathbb{P}(V), \mathbb{Z} \mathfrak{S}) \rightarrow E_{2 k, 2}^{\infty} \rightarrow H_{2 k+2}\left(\mathbb{P}\left(p_{1}^{*}\left(\Theta_{U}\right) \oplus p_{2}^{*}\left(\Theta_{V}\right)\right), \mathbb{Z}\right) .
$$

We define the morphism $J_{U V}: H_{2 k}(\mathbb{P}(U) \times \mathbb{P}(V), \mathbb{Z}) \rightarrow H_{2 k+2}(\mathbb{P}(U \oplus V), \mathbb{Z})$ by

$$
J_{U V}=\left(q_{U V}\right)_{*} \circ \sigma_{U V}
$$

The morphism $\sigma_{U V}$ can be geometrically understood as follows. Let $M, N$ be closed, connected oriented manifolds and $\varphi: M \rightarrow \mathbb{P}(U), \psi: N \rightarrow \mathbb{P}(V)$ smooth maps. The manifold $P_{\varphi, \psi}:=\mathbb{P}\left(\varphi^{*}\left(\Theta_{U}\right) \oplus \psi^{*}\left(\Theta_{V}\right)\right)$ is a $\mathbb{P}_{\mathbb{C}}^{1}$ fiber bundle over $M \times N$, hence it comes with a canonical orientation induced by the complex orientations of the fibers and and the product orientation of the basis. Then

Remark 3.5. The class $\sigma_{U V}\left(\varphi_{*}[M] \otimes \psi_{*}[N]\right)$ coincides with the image of the fundamental class $\left[P_{\varphi, \psi}\right]$ under the natural map $P_{\varphi, \psi} \rightarrow \mathbb{P}\left(p_{1}^{*}\left(\Theta_{U}\right) \oplus p_{2}^{*}\left(\Theta_{V}\right)\right)$.

Using Remark 3.5 one can check that the definition (13) is compatible with set theoretical join map defined above restricted to the set of algebraic subvarieties of $\mathbb{P}(U) \times \mathbb{P}(V)$. More precisely, for an algebraic subvariety $A \subset \mathbb{P}(U) \times \mathbb{P}(V)$, one has

$$
J_{U V}([A])=\left[q_{U V}\left(p_{U V}^{-1}(A)\right)\right],
$$

where [ ] stands for the fundamental class of an analytic cycle. This gives

Remark 3.6. Using the Küneth isomorphism $H^{*}(\mathbb{P}(U) \times \mathbb{P}(V), \mathbb{Z})=H^{*}(\mathbb{P}(U), \mathbb{Z}) \otimes$ $H^{*}(\mathbb{P}(V), \mathbb{Z})$ and denoting by $u_{k}, v_{k}, w_{k}$ the fundamental class of the $k$-dimensional linear variety in $\mathbb{P}(U), \mathbb{P}(V), \mathbb{P}(U \oplus V)$ respectively, we have

$$
J_{U V}\left(u_{k} \otimes v_{l}\right)=w_{k+l+1} .
$$

We need a version of the join morphism which takes values in $H_{*}(\mathbb{P}(U \oplus \bar{V}), \mathbb{Z})$. We denote by $\bar{V}$ the $\mathbb{C}$-vector space obtained by endowing $V$ with the scalar multiplication $\zeta \cdot \bar{V} v:=\bar{\zeta} \cdot V v$. The identity map $V \rightarrow \bar{V}$ becomes anti-linear and will be denoted by $v \mapsto \bar{v}$. For a subset $A \subset \mathbb{P}(U) \times \mathbb{P}(V)$ we define its set theoretical join $J_{U}^{V}(A) \subset \mathbb{P}(U \oplus \bar{V})$ by

$$
J_{U}^{V}(A):=\bigcup_{([u],[v]) \in A} \mathbb{P}(\mathbb{C} u \oplus \mathbb{C} \bar{v}) \subset \mathbb{P}(U \oplus \bar{V}) .
$$

Correspondingly, we replace in diagram (11) 
- the projective line bundle $p_{U V}: \mathbb{P}\left(p_{1}^{*}\left(\Theta_{U}\right) \oplus p_{1}^{*}\left(\Theta_{V}\right)\right) \rightarrow \mathbb{P}(U) \times \mathbb{P}(V)$ by

$$
p_{U}^{V}: \mathbb{P}\left(p_{1}^{*}\left(\Theta_{U}\right) \oplus p_{1}^{*}\left(\bar{\Theta}_{V}\right)\right) \rightarrow \mathbb{P}(U) \times \mathbb{P}(V),
$$

where $\bar{\Theta}_{V}$ is the line bundle $V^{*} \times_{\bar{\chi}_{0}^{-1}} \mathbb{C}^{*}$ on $\mathbb{P}(V)$. Therefore

$$
\mathbb{P}\left(p_{1}^{*}\left(\Theta_{U}\right) \oplus p_{1}^{*}\left(\bar{\Theta}_{V}\right)\right)=\left(U^{*} \times V^{*}\right) \times_{\alpha^{\prime}} \mathbb{P}^{1},
$$

where $\alpha^{\prime}:\left(\mathbb{C}^{*} \times \mathbb{C}^{*}\right) \times \mathbb{P}^{1} \rightarrow \mathbb{P}^{1}$ acts by:

$$
\alpha^{\prime}\left(\left(\zeta_{1}, \zeta_{2}\right),\left[z_{0}, z_{1}\right]\right)=\left[\zeta_{1}^{-1} z_{0}, \bar{\zeta}_{2}^{-1} z_{1}\right]
$$

- the map $q_{U V}$ by the map

$$
\begin{gathered}
q_{U}^{V}: \mathbb{P}\left(p_{1}^{*}\left(\Theta_{U}\right) \oplus p_{1}^{*}\left(\bar{\Theta}_{V}\right)\right) \rightarrow \mathbb{P}(U \oplus \bar{V}), \\
q_{U}^{V}\left(\left[(u, v),\left[z_{0}, z_{1}\right]\right]\right):=\left[z_{0} u, z_{1} \bar{v}\right] \forall(u, v) \in U^{*} \times V^{*}, \forall\left[z_{0}, z_{1}\right] \in \mathbb{P}_{\mathbb{C}}^{1} .
\end{gathered}
$$

In this way we obtain a homological version of (14), namely

$$
J_{U}^{V}=q_{U *}^{V} \circ \sigma_{U}^{V}: H_{2 k}(\mathbb{P}(U) \times \mathbb{P}(V), \mathbb{Z}) \rightarrow H_{2 k+2}(\mathbb{P}(U \oplus \bar{V}), \mathbb{Z}),
$$

where

$$
\sigma_{U}^{V}: H_{2 k}(\mathbb{P}(U) \times \mathbb{P}(V), \mathbb{Z}) \rightarrow H_{2 k+2}\left(\mathbb{P}\left(p_{1}^{*}\left(\Theta_{U}\right) \oplus p_{2}^{*}\left(\bar{\Theta}_{V}\right)\right), \mathbb{Z}\right)
$$

is obtained in the same way as $\sigma_{U V}$, but using the Gysin exact sequence of the sphere bundle $p_{U}^{V}$.

Denoting by $I_{U}^{V}: \mathbb{P}(U) \times \mathbb{P}(V) \rightarrow \mathbb{P}(U) \times \mathbb{P}(\bar{V})$ the homeomorphism given by $([u],[v]) \mapsto([u],[\bar{v}])$, we have the identity

$$
J_{U}^{V}=J_{U \bar{V}} \circ\left(I_{U}^{V}\right)_{*},
$$

which yields following version of Remark 3.6

Remark 3.7. Denoting by $u_{k}, v_{k}, w_{k}$ the fundamental class of the $k$-dimensional linear variety in $\mathbb{P}(U), \mathbb{P}(V), \mathbb{P}(U \oplus \bar{V})$ respectively, and using the Küneth isomorphism we have

$$
J_{U}^{V}\left(u_{k} \otimes v_{l}\right)=(-1)^{l} w_{k+l+1} .
$$

3.3. The closure in $\hat{Q}_{f}$ of an analytic set $Z \subset Q_{f}^{s}$ and the boundary of its fundamental class. We come back to the blow up flip passage $\hat{Q}_{f}$ associated with the data $\left(p^{\prime}: E^{\prime} \rightarrow B, p^{\prime \prime}: E^{\prime \prime} \rightarrow B, f\right)$ as explained in section 1 .

Let $Z \subset Q_{f}^{s}$ be an analytic set of pure dimension $k>0$, and let $[Z]^{B M} \in$ $H_{2 k}^{B M}\left(Q_{f}^{s}, \mathbb{Z}\right)$ be the fundamental class of $Z$ in the Borel-Moore homology of $Q_{f}^{s}$. The long exact sequence associated to the open embedding $Q_{f}^{s} \hookrightarrow \hat{Q}_{f}$ contains the segment

$$
\cdots \rightarrow H_{2 k}^{B M}\left(\hat{Q}_{f}, \mathbb{Z}\right) \rightarrow H_{2 k}^{B M}\left(Q_{f}^{s}, \mathbb{Z}\right) \stackrel{\delta}{\rightarrow} H_{2 k-1}^{B M}\left(\partial \hat{Q}_{f}, \mathbb{Z}\right) \rightarrow \ldots
$$

We will give an explicit formula, in complex geometric terms, for the boundary

$$
\delta\left([Z]^{B M}\right) \in H_{2 k-1}^{B M}\left(\partial \hat{Q}_{f}, \mathbb{Z}\right)=H_{2 k-1}\left(\mathbb{P}\left(F^{\prime} \oplus \bar{F}^{\prime \prime}\right), \mathbb{Z}\right) .
$$

The problem is difficult because, a priori, one has no control on the closure of $Z$ in the manifold with boundary $\hat{Q}_{f}$. Our result will implicitly give an explicit construction of this closure, and show that it belongs to a very special class of real analytic sets of $\hat{Q}_{f}$.

We recall that the circle $C=f^{-1}(0)$ has been endowed with the orientation defined by the complex orientation of $B$ and the orientation of $N_{C}^{B}$ defined by the differential $d f$. Equivalently, $C$ is endowed with the boundary orientation of 
$\partial f^{-1}([0, \infty))$. The bundles $F^{\prime}, F^{\prime \prime}$ on $C$ are trivial hence, choosing a point $x \in C$ we get a canonical isomorphism $H_{*}\left(\mathbb{P}\left(F^{\prime} \oplus \bar{F}^{\prime \prime}\right), \mathbb{Z}\right)=H_{*}(C, \mathbb{Z}) \otimes H_{*}\left(\mathbb{P}\left(F_{x}^{\prime} \oplus \bar{F}_{x}^{\prime \prime}\right), \mathbb{Z}\right)$. In particular, for $1 \leq k \leq r^{\prime}+r^{\prime \prime}$ one has

$$
H_{2 k-1}\left(\mathbb{P}\left(F^{\prime} \oplus \bar{F}^{\prime \prime}\right), \mathbb{Z}\right)=[C] \otimes \mathbb{Z} w_{k-1},
$$

where $w_{k-1}$ denotes the standard generator of $H_{2 k-2}\left(\mathbb{P}\left(F_{x}^{\prime} \oplus \bar{F}_{x}^{\prime \prime}\right), \mathbb{Z}\right)$.

Note first that, by definition, $Q_{f}^{s}$ contains two obvious complex submanifolds for which the problem can be solved easily: Putting $P^{\prime}:=\mathbb{P}\left(E^{\prime}\right), P^{\prime \prime}:=\mathbb{P}\left(E^{\prime \prime}\right)$, $B_{ \pm}:=( \pm f)^{-1}(0, \infty), E_{ \pm}^{\prime}:=\left.E^{\prime}\right|_{B_{ \pm}}, E_{ \pm}^{\prime \prime}:=\left.E^{\prime \prime}\right|_{B_{ \pm}}, P_{ \pm}^{\prime}:=\left.P^{\prime}\right|_{B_{ \pm}}, P_{ \pm}^{\prime \prime}:=\left.P^{\prime \prime}\right|_{B_{ \pm}}$(as in section 1), we see that $P_{-}^{\prime}, P_{+}^{\prime \prime}$ are analytic subsets of $Q_{f}^{s}$.

Remark 3.8. Under the assumptions and with the notations above one has

$\delta\left(\left[P_{-}^{\prime}\right]^{B M}\right)=-[C] \otimes w_{r^{\prime}-1}$ if $r^{\prime}>0, \delta\left(\left[P_{+}^{\prime \prime}\right]^{B M}\right)=(-1)^{r^{\prime \prime}-1}[C] \otimes w_{r^{\prime \prime}-1}$ if $r^{\prime \prime}>0$.

The first (second) formula also applies in the special case when $r^{\prime}=0\left(r^{\prime \prime}=0\right)$; in this special case we have $P_{-}^{\prime}=\emptyset, P_{+}^{\prime \prime}=Q_{f}^{s}$ (respectively $P_{+}^{\prime \prime}=\emptyset, P_{-}^{\prime}=Q_{f}^{s}$ ).

The union $P_{-}^{\prime} \cup P_{+}^{\prime \prime}$ is disjoint, and should be regarded as the known part of $Q_{f}^{s}$. The main result of this section concerns the more difficult case of a pure $k$ dimensional analytic set $Z$ which is generically exterior to this "known" part of $Q_{f}^{s}$, in the sense that $\operatorname{dim}\left(Z \cap\left(P_{-}^{\prime} \cup P_{+}^{\prime \prime}\right)\right)<k$.

Let $E_{*}^{\prime}, E_{*}^{\prime \prime}$ be the complements of the zero sections in the bundles $E^{\prime}, E^{\prime \prime}$. Regarding $E_{*}^{\prime} \times_{B} E_{*}^{\prime \prime}$ as a left principal $\mathbb{C}^{*} \times \mathbb{C}^{*}$-bundle over $P^{\prime} \times_{B} P^{\prime \prime}$, consider the associated line bundle given by

$$
q: Q:=\left(E_{*}^{\prime} \times_{B} E_{*}^{\prime \prime}\right) \times\left(\chi_{1}^{-1} \chi_{2}^{-1}\right) \mathbb{C} \longrightarrow P^{\prime} \times_{B} P^{\prime \prime},
$$

where $\chi_{i}: \mathbb{C}^{*} \times \mathbb{C}^{*} \rightarrow \mathbb{C}^{*}$ is the character defined by the projection on the $i$-th factor. In other words, denoting by $\Theta^{\prime}, \Theta^{\prime \prime}$ the tautological line bundles on the projective bundles $P^{\prime}, P^{\prime \prime}$ and by $p_{1}: P^{\prime} \times_{B} P^{\prime \prime} \rightarrow P^{\prime}, p_{2}: P^{\prime} \times_{B} P^{\prime \prime} \rightarrow P^{\prime \prime}$ the two projections, one has $Q=p_{1}^{*}\left(\Theta^{\prime}\right) \otimes p_{2}^{*}\left(\Theta^{\prime \prime}\right)$ as line bundles over $P^{\prime} \times{ }_{B} P^{\prime \prime}$. The zero section of this line bundle is a smooth hypersurface which can be identified with $P^{\prime} \times_{B} P^{\prime \prime}$.

We denote by $Q_{0} \subset E^{\prime} \otimes E^{\prime \prime}$ the image of the natural map $E^{\prime} \times_{B} E^{\prime \prime} \rightarrow E^{\prime} \otimes E^{\prime \prime}$. This map is obviously $\mathbb{C}^{*}$-invariant. $Q_{0}$ is a locally trivial fiber bundle over $B$ whose fiber over $x \in B$ is the cone over the image of $P_{x}^{\prime} \times P_{x}^{\prime \prime}$ via the Segre embedding $P_{x}^{\prime} \times P_{x}^{\prime \prime} \rightarrow \mathbb{P}\left(E_{x}^{\prime} \otimes E_{x}^{\prime \prime}\right)$. This cone is singular when $r^{\prime}>1$ and $r^{\prime \prime}>1$, hence in this case $Q_{0}$ is a singular complex space whose singular locus is the zero section $B \subset E^{\prime} \otimes E^{\prime \prime}$. One has an obvious contraction $c: Q \rightarrow Q_{0}$ which induces a biholomorphism $Q \backslash\left(P^{\prime} \times{ }_{B} P^{\prime \prime}\right) \rightarrow Q_{0} \backslash B$, and contracts fiberwise the divisor $P^{\prime} \times{ }_{B} P^{\prime \prime}$ to the zero section $B$ of $Q_{0}$. In other words $c$ is the modification with center $B$, and the fiber product $P^{\prime} \times_{B} P^{\prime \prime}$ is the exceptional divisor of the modification $c$. In the diagram below all polygons are commutative, $j_{1}, j_{2}$ are biholomorphisms. The maps $c_{1}, c_{2}$ are holomorphic modifications, in particular they are proper. The map $c_{1}$ contracts $P_{x}^{\prime} \times P_{x}^{\prime \prime}$ to $P_{x}^{\prime}$ when $x \in B_{-}$and contracts $P_{x}^{\prime} \times P_{x}^{\prime \prime}$ to $P_{x}^{\prime \prime}$ when $x \in B_{+}$. The map $c_{2}$ contacts $P_{x}^{\prime} \subset Q_{f}^{s}$ to $\{x\}$ when $x \in B_{-}$, and contacts $P_{x}^{\prime \prime} \subset Q_{f}^{s}$ to $\{x\}$ when $x \in B_{+}$. Note that

$$
c_{2} \circ c_{1}=c_{\mid Q \backslash\left(P_{C}^{\prime} \times{ }_{C} P_{C}^{\prime \prime}\right)} .
$$




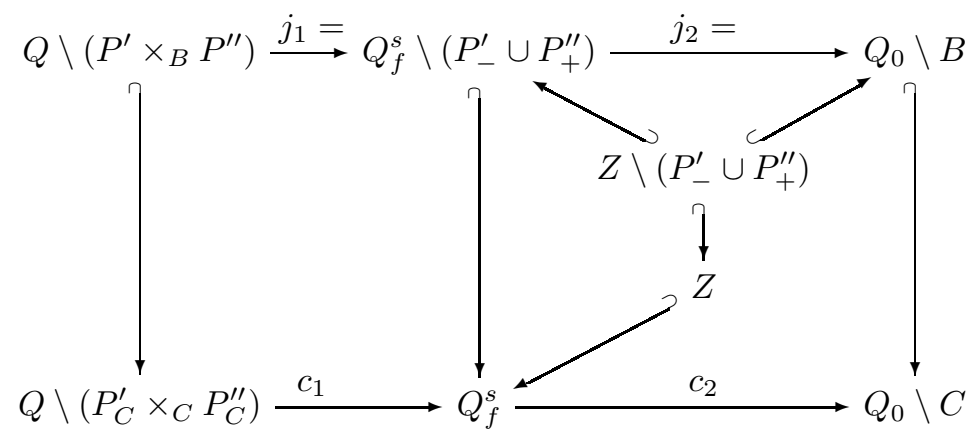

Using the notations introduced in sections 3.13 .2 we can state now

Theorem 3.9. With the notations above, suppose $r^{\prime}>0, r^{\prime \prime}>0$, and let $Z \subset Q_{f}^{s}$ be an analytic subset of pure dimension $k \geq 1$, such that $\operatorname{dim}\left(Z \cap\left(P_{-}^{\prime} \cup P_{+}^{\prime \prime}\right)\right)<k$. Then

(1) The closure $\tilde{Z}$ of $j_{1}^{-1}\left(Z \backslash\left(P_{-}^{\prime} \cup P_{+}^{\prime \prime}\right)\right)$ in $Q$ is a pure $k$-dimensional analytic subset of $Q$ with $\operatorname{dim}\left(\tilde{Z} \cap\left(P^{\prime} \times{ }_{B} P^{\prime \prime}\right)\right)<k$,

(2) Choosing a point $x \in C$, the equality

$$
\begin{gathered}
\delta[Z]^{B M}=[C] \otimes J_{E_{x}^{\prime}}^{E_{x}^{\prime \prime}}\left([\tilde{Z}]^{B M} \cdot\left(P_{x}^{\prime} \times P_{x}^{\prime \prime}\right)\right) \\
\text { holds in } H_{1}(C, \mathbb{Z}) \otimes H_{2 k-2}\left(\mathbb{P}\left(F_{x}^{\prime} \oplus \bar{F}_{x}^{\prime \prime}\right), \mathbb{Z}\right)=H_{2 k-1}\left(\mathbb{P}\left(F^{\prime} \oplus \bar{F}^{\prime \prime}\right), \mathbb{Z}\right) .
\end{gathered}
$$

The second statement gives an explicit formula for the boundary $\delta[Z]^{B M}$ in complex geometric terms: this class is determined by the homological intersection (see Definition [3.1) of the Borel Moore class of $[\tilde{Z}]$ with the complex submanifold $P_{x}^{\prime} \times P_{x}^{\prime \prime}$ of $Q$.

Proof. (1) Since we assumed $\operatorname{dim}\left(Z \cap\left(P_{-}^{\prime} \cup P_{+}^{\prime \prime}\right)\right)<k$, it follows that $Z$ coincides with the closure of $Z \backslash\left(P_{-}^{\prime} \cup P_{+}^{\prime \prime}\right)$ in $Q_{f}^{s}$. We will apply Remmert-Stein theorem [GR, sec. 2 p. 354] to the inclusion

$$
j_{2}\left(Z \backslash\left(P_{-}^{\prime} \cup P_{+}^{\prime \prime}\right)\right) \hookrightarrow Q_{0} \backslash B .
$$

This theorem applies automatically when $k \geq 2$ and shows that the closure $Z_{0}$ of $j_{2}\left(Z \backslash\left(P_{-}^{\prime} \cup P_{+}^{\prime \prime}\right)\right)$ in $Q_{0}$ is a complex analytic set of dimension $k$. In the limit case $k=1=\operatorname{dim}(B)$ we have to check that this closure $Z_{0}$ does not contain all of $B$. Since, by assumption, $\operatorname{dim}\left(Z \cap\left(P_{-}^{\prime} \cup P_{+}^{\prime \prime}\right)\right)<1$, it follows that $A:=Z \cap\left(P_{-}^{\prime} \cup P_{+}^{\prime \prime}\right)$ is a 0 -dimensional analytic subset of $P_{-}^{\prime} \cup P_{+}^{\prime \prime}$. We claim that

$$
Z_{0} \cap(B \backslash C) \subset c_{2}(A),
$$

which will complete the argument. To prove this inclusion, let $x \in B \backslash C$ be the limit (in $\left.Q_{0}\right)$ of a sequence $\left(j_{2}\left(z_{n}\right)\right)_{n \in \mathbb{N}}$ of points of $j_{2}\left(Z \backslash\left(P_{-}^{\prime} \cup P_{+}^{\prime \prime}\right)\right)$. Since $c_{2}$ is proper, a subsequence $\left(z_{n_{k}}\right)_{k \in \mathbb{N}}$ of $\left(z_{n}\right)_{n \in \mathbb{N}}$ will converge to a point in $y \in P_{-}^{\prime} \cup P_{+}^{\prime \prime}$ with $c_{2}(y)=x$. But $Z$ is closed in $Q_{f}^{s}$, hence we will have $y \in Z$, hence $x \in c_{2}(A)$ as claimed.

Since $c$ is a modification, the closure $\tilde{Z}$ of $c^{-1}\left(Z_{0} \backslash B\right)=j_{1}^{-1}\left(Z \backslash\left(P_{-}^{\prime} \cup P_{+}^{\prime \prime}\right)\right)$ in $Q$ is an analytic set. This analytic set is the proper transform $\tilde{Z}$ of $Z_{0}$ in $Q$, and it is pure $k$-dimensional, because $Z_{0} \backslash B$ has this property. Note also that $\operatorname{dim}\left(\tilde{Z} \cap\left(P^{\prime} \times{ }_{B} P^{\prime \prime}\right)\right)<k$ as claimed, because the complement of $\tilde{Z} \cap\left(P^{\prime} \times{ }_{B} P^{\prime \prime}\right)$ in $\tilde{Z}$ (which is obviously Zariski open in $\tilde{Z}$ ) is dense in $\tilde{Z}$. 
(2) Denote by $P_{C}^{\prime}, P_{C}^{\prime \prime}$ the restrictions of $P^{\prime}, P^{\prime \prime}$ to $C$, and put $W:=P_{C}^{\prime} \times_{C} P_{C}^{\prime \prime}$ regarded a submanifold of $Q$ of real codimension 3. As a subset of the smooth hypersurface $P^{\prime} \times_{B} P^{\prime \prime} \subset Q$ (the zero section of $Q$ ), $W$ is the smooth real hypersurface defined by the equation $f \circ \pi=0$, where $\pi: P^{\prime} \times_{B} P^{\prime \prime} \rightarrow B$ is the obvious projection. The idea of the proof is to construct a continuous extension $\hat{c}: \hat{Q}_{W} \rightarrow \hat{Q}_{f}$ of $c_{1}$ and to make use of Proposition 3.3 applied to $\left(Q, W,[\tilde{Z}]^{B M}\right)$ in order to compute the boundary of $[\tilde{Z} \backslash W]^{B M}$ in the homology of $\partial \hat{Q}_{W}$.

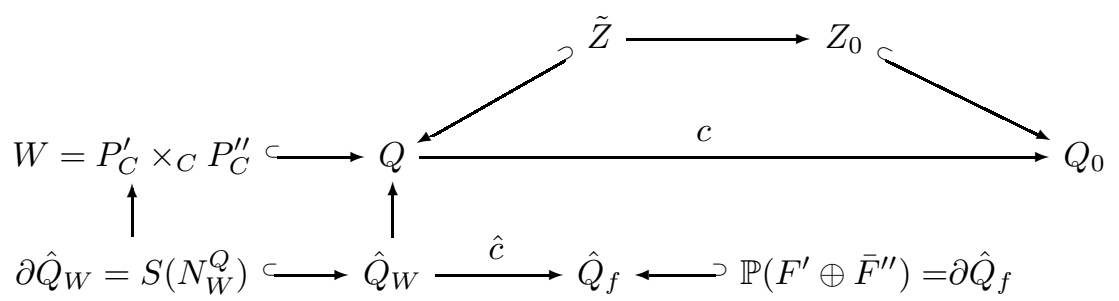

Note first that $W$ is the zero locus of the section $\left(\theta_{Q}, f \circ \pi \circ q\right)$ in $q^{*}(Q) \oplus \underline{\mathbb{R}}$, where $\theta_{Q}$ is the tautological section of the complex line bundle $q^{*}(Q)$ over $Q$. Therefore, as explained section 2.1 the blow up $\hat{Q}_{W}$ can be identified with the submanifold

$$
\{((y, t), \rho) \in S(Q \oplus \underline{\mathbb{R}}) \times[0, \infty) \mid \rho t=f(\pi(q(y)))\}
$$

of $S(Q \oplus \underline{\mathbb{R}}) \times[0, \infty)$, and, via this identification, the contraction map $\hat{Q}_{W} \rightarrow Q$ is given by $((y, t), \rho) \mapsto \rho y$. Note that the sphere bundle $S(Q \oplus \underline{\mathbb{R}})$ is the associated bundle

$$
\left(S\left(E^{\prime}\right) \times_{B} S\left(E^{\prime \prime}\right)\right) \times_{\beta} S(\mathbb{C} \oplus \mathbb{R}),
$$

where $S\left(E^{\prime}\right) \times{ }_{B} S\left(E^{\prime \prime}\right)$ is regarded as a left principal $S^{1} \times S^{1}$-bundle over $P^{\prime} \times{ }_{B} P^{\prime \prime}$, and the action $\beta:\left(S^{1} \times S^{1}\right) \times S(\mathbb{C} \oplus \mathbb{R}) \rightarrow S(\mathbb{C} \oplus \mathbb{R})$ is given by

$$
\beta\left(\left(\zeta_{1}, \zeta_{2}\right),(u, t)\right)=\left(\zeta_{1}^{-1} \zeta_{2}^{-1} u, t\right) .
$$

Using formula (3) of section 2.1. one has an identification

$$
\hat{Q}_{f}=\left\{\left(\left[y^{\prime}, \bar{y}^{\prime \prime}\right], \rho\right) \in\left\{S\left(E^{\prime} \oplus \bar{E}^{\prime \prime}\right) / S^{1}\right\} \times[0, \infty) \mid m^{f}\left(\rho y^{\prime}, \rho y^{\prime \prime}\right)=0\right\},
$$

and, via this identification, the contraction map $\hat{Q}_{f} \rightarrow Q_{f}$ is $\left(\left[y^{\prime}, y^{\prime \prime}\right], \rho\right) \rightarrow\left[\rho y^{\prime}, \rho y^{\prime \prime}\right]$.

We define $c_{f}: \hat{Q}_{W} \rightarrow \hat{Q}_{f}$ by

$$
\hat{c}\left(\left[\left(a^{\prime}, a^{\prime \prime}\right),(u, t)\right], \rho\right):=\left\{\begin{array}{lll}
\left(\frac{1}{\sqrt{2}}\left[(1-t)^{\frac{1}{2}} a^{\prime},(1-t)^{-\frac{1}{2}} \bar{u} \bar{a}^{\prime \prime}\right], \sqrt{2 \rho}\right) & \text { for } t \neq 1 \\
\left(\frac { 1 } { \sqrt { 2 } } \left[u(1+t)^{-\frac{1}{2}}\right.\right. & \left.\left.a^{\prime},(1+t)^{\frac{1}{2}} \bar{a}^{\prime \prime}\right], \sqrt{2 \rho}\right) & \text { for } t \neq-1
\end{array},,\right.
$$

for any $\left(a^{\prime}, a^{\prime \prime}\right) \in S\left(E^{\prime}\right) \times_{B} S\left(E^{\prime \prime}\right),(u, t) \in S(\mathbb{C} \oplus \mathbb{R}), \rho \in[0, \infty)$. It is easy to see that $\hat{c}$ is well defined, takes values in $\hat{Q}_{f}$, and is a continuous extension of the holomorphic modification $c_{1}$.

The boundary of $[\tilde{Z} \backslash W]^{B M}$ in $H_{2 k-1}\left(\partial \hat{Q}_{W}, \mathbb{Z}\right)$ can be computed easily using Proposition 3.3 and the result is:

$$
\delta\left([\tilde{Z} \backslash W]^{B M}\right)=\left([\tilde{Z}]^{B M} \cdot W\right) \otimes \mathfrak{S}_{N_{W}^{Q}},
$$

where $[\tilde{Z}]^{B M} \cdot W \in H_{2 k-3}(W, \mathbb{Z})$ is given by Definition 3.1 and $\mathfrak{S}_{N_{W}^{Q}}$ is the section defined by the fundamental classes of the spheres $S\left(N_{W, w}^{Q}\right), w \in W$. In this formula $N_{W}^{Q}$ is oriented using the decomposition $N_{W}^{Q}=\left.Q\right|_{W} \oplus \underline{\mathbb{R}}$, and the complex orientation of the complex line bundle $Q$. Correspondingly, $W$ is oriented using this 
orientation of $N_{W}^{Q}$, and the complex orientation of the manifold $Q$. This orientation of $W$ agrees with its orientation as $\mathbb{P}_{\mathbb{C}}^{r^{\prime}} \times \mathbb{P}_{\mathbb{C}}^{r^{\prime \prime}}$-bundle over the oriented circle $C$.

Under our assumptions $W$ is a $\mathbb{P}_{\mathbb{C}}^{r^{\prime}} \times \mathbb{P}_{\mathbb{C}}^{r^{\prime \prime}}$ bundle over $C$ (which is a circle), hence any cohomology class $b$ of even degree of $W$ can be written as $1 \otimes j_{x}^{*}(b)$, where $j_{x}: P_{x}^{\prime} \times P_{x}^{\prime \prime} \hookrightarrow W$ denotes the inclusion map. Therefore, for any Borel-More homology class $c$ of even degree of $Q$, denoting by $b$ its Poincaré dual, we get

$c \cdot W=j_{W}^{*}(b) \cap[W]=\left(1 \otimes j_{x}^{*} j_{W}^{*}(b)\right) \cap\left([C] \otimes\left[P_{x}^{\prime} \times P_{x}^{\prime \prime}\right]\right)=[C] \otimes\left(c \cdot\left(P_{x}^{\prime} \times P_{x}^{\prime \prime}\right)\right)$.

In particular

$$
[\tilde{Z}]^{B M} \cdot W=[C] \otimes\left([\tilde{Z}]^{B M} \cdot\left(P_{x}^{\prime} \times P_{x}^{\prime \prime}\right)\right)
$$

so that, by (22) we get

$$
\delta\left([\tilde{Z} \backslash W]^{B M}\right)=[C] \otimes\left([\tilde{Z}]^{B M} \cdot\left(P_{x}^{\prime} \times P_{x}^{\prime \prime}\right)\right) \otimes \mathfrak{S}_{N_{W}^{Q}} .
$$

The morphism of triples $\left(\hat{Q}_{W} \backslash \partial \hat{Q}_{W}, \hat{Q}_{W}, \partial \hat{Q}_{W}\right) \rightarrow\left(\hat{Q}_{f} \backslash \partial \hat{Q}_{f}, \hat{Q}_{f}, \partial \hat{Q}_{f}\right)$ induced by $\hat{c}$ gives a morphism of Borel-Moore homology long exact sequences. Since

$$
c_{1} \mid \tilde{Z} \backslash W: \tilde{Z} \backslash W \rightarrow Z
$$

is a modification, we have $\left(c_{1 *}\right)\left([\tilde{Z} \backslash W]^{B M}\right)=[Z]^{B M}$, which implies

$$
\delta\left([Z]^{B M}\right)=(\partial \hat{c})_{*}\left(\delta\left([\tilde{Z} \backslash W]^{B M}\right)\right) .
$$

The two boundaries $\partial \hat{Q}_{W}, \partial \hat{Q}_{f}$ are fiber bundles over $C$, and the map

$$
\partial \hat{c}_{x}:\left(S\left(E_{x}^{\prime}\right) \times S\left(E_{x}^{\prime \prime}\right)\right) \times_{\beta} S(\mathbb{C} \oplus \mathbb{R}) \rightarrow \mathbb{P}\left(E_{x}^{\prime} \oplus \bar{E}_{x}^{\prime \prime}\right)
$$

induced by $\partial \hat{c}$ is a bundle morphism over $C$. Therefore, writing $\delta\left([Z]^{B M}\right)$ as $\delta\left([Z]^{B M}\right)=[C] \otimes U$ for a class $U \in H_{2 k-2}\left(\mathbb{P}\left(E_{x}^{\prime} \oplus \bar{E}_{x}^{\prime \prime}\right), \mathbb{Z}\right)$ we get

$$
U=\left(\partial \hat{c}_{x}\right)_{*}\left(\left([\tilde{Z}]^{B M} \cdot\left(P_{x}^{\prime} \times P_{x}^{\prime \prime}\right)\right) \otimes \mathfrak{S}_{N_{W}^{Q}}\right) .
$$

Therefore to complete the proof it suffices to show that

$$
\left(\partial \hat{c}_{x}\right)_{*}\left(\left([\tilde{Z}]^{B M} \cdot\left(P_{x}^{\prime} \times P_{x}^{\prime \prime}\right)\right) \otimes \mathfrak{S}_{N_{W}^{Q}}\right)=J_{E_{x}^{\prime \prime}}^{E_{x}^{\prime \prime}}\left([\tilde{Z}]^{B M} \cdot\left(P_{x}^{\prime} \times P_{x}^{\prime \prime}\right)\right) .
$$

Taking into account (21) the map $\partial \hat{c}_{x}$ decomposes as $\partial \hat{c}_{x}=q_{x} \circ G_{x}$, where

- $G_{x}:\left(S\left(E_{x}^{\prime}\right) \times S\left(E_{x}^{\prime \prime}\right)\right) \times{ }_{\beta} S(\mathbb{C} \oplus \mathbb{R}) \rightarrow\left(S\left(E_{x}^{\prime}\right) \times S\left(E_{x}^{\prime \prime}\right)\right) \times{ }_{\alpha^{\prime}} \mathbb{P}_{\mathbb{C}}^{1}$ is the bundle isomorphism induced by the diffeomorphism $g: S(\mathbb{C} \oplus \mathbb{R}) \rightarrow \mathbb{P}_{\mathbb{C}}^{1}$ given by

$g(u, t)=\left\{\begin{array}{l}{\left[(1-t)^{\frac{1}{2}}, \bar{u}(1-t)^{-\frac{1}{2}}\right] \text { for } t \neq 1} \\ {\left[u(1+t)^{-\frac{1}{2}},(1+t)^{\frac{1}{2}}\right] \text { for } t \neq-1}\end{array} \forall(u, t) \in S(\mathbb{C} \oplus \mathbb{R})\right.$.

This diffeomorphism is orientation preserving if $S(\mathbb{C} \oplus \mathbb{R})$ is endowed with the standard orientation (as boundary of $D^{3}$ ) and $\mathbb{P}_{\mathbb{C}}^{1}$ with the complex orientation; it maps $\left(0_{\mathbb{C}}, 1\right)$ to $\infty \in \mathbb{P}_{\mathbb{C}}^{1},\left(0_{\mathbb{C}},-1\right)$ to $0 \in \mathbb{P}_{\mathbb{C}}^{1}$, and is equivariant with respect to the $S^{1} \times S^{1}$-actions $\beta, \alpha^{\prime}$ given by the formulae (20), (16).

- $q_{x}:\left(S\left(E_{x}^{\prime}\right) \times S\left(E_{x}^{\prime \prime}\right)\right) \times_{\alpha^{\prime}} \mathbb{P}_{\mathbb{C}}^{1} \rightarrow \mathbb{P}\left(E_{x}^{\prime} \oplus \bar{E}_{x}^{\prime \prime}\right)$ is defined by

$$
q_{x}\left(\left[\left(a^{\prime}, a^{\prime \prime}\right),\left[z_{0}, z_{1}\right]\right]\right):=\left[z_{0} a^{\prime}, z_{1} \bar{a}^{\prime \prime}\right] .
$$

It suffices to recall that, by (15), one has

$$
\left(S\left(E_{x}^{\prime}\right) \times S\left(E_{x}^{\prime \prime}\right)\right) \times_{\alpha^{\prime}} \mathbb{P}_{\mathbb{C}}^{1}=\mathbb{P}\left(p_{1}^{*}\left(\Theta_{E_{x}^{\prime}}\right) \oplus p_{2}^{*}\left(\bar{\Theta}_{E_{x}^{\prime \prime}}\right),\right.
$$

and to note that, via this identification, one has $q_{x}=q_{E_{x}^{\prime \prime}}^{E_{x}^{\prime \prime}}$. 
Using our explicit formula for the join morphism (see Remark 3.7), Proposition 3.9, and Remark 3.8, we get

Corollary 3.10. With the notations introduced above, suppose $r^{\prime}+r^{\prime \prime}>0$. The boundary of the fundamental class $\left[Q_{f}^{s}\right]^{B M}$ of the whole quotient $Q_{f}^{s}$ is given by

$$
\delta\left(\left[Q_{f}^{s}\right]^{B M}\right)=(-1)^{r^{\prime \prime}-1}[C] \otimes w_{r^{\prime}+r^{\prime \prime}-1}=(-1)^{r^{\prime \prime}-1}[C] \otimes\left[\mathbb{P}\left(E_{x}^{\prime} \oplus \bar{E}_{x}^{\prime \prime}\right)\right] .
$$

Note that this formula holds even when $r^{\prime \prime}=0$, or $r^{\prime}=0$, but in these cases one uses Remark 3.8. When $r^{\prime \prime}=0\left(r^{\prime}=0\right)$, one has $Q_{f}^{s}=P_{-}^{\prime}$ (respectively $\left.Q_{f}^{s}=P_{+}^{\prime \prime}\right)$.

Example 3.2. Suppose $r^{\prime \prime}=1$. Then

$$
\left.\left.\delta\left(\left[P_{-}^{\prime}\right]^{B M}\right)\right)=-[C] \otimes w_{1}, \delta\left(\left[P_{+}^{\prime \prime}\right]^{B M}\right)\right)=[C] \otimes 1, \delta\left(\left[Q_{f}^{s}\right]^{B M}\right)=[C] \otimes w_{2}
$$

In this case $Q_{0}$ can be identified with $E^{\prime} \otimes E^{\prime \prime}$ (hence is smooth), $Q$ is the blow up of $Q_{0}$ at the curve $B, Q_{f}^{\text {st }}$ is the blow up of $Q_{0} \backslash C$ at the curve $B_{-}, c: Q \rightarrow Q_{0}$, $c_{2}: Q_{f}^{\text {st }} \rightarrow Q_{0} \backslash C$ are the corresponding contraction maps, the projective bundles $P^{\prime}, P_{-}^{\prime}$ are the corresponding exceptional divisors, and $P_{+}^{\prime \prime}=B_{+}$. Let $Z \subset Q_{f}^{s}$ be an irreducible hypersurface. Taking into account the position of $Z$ with respect to $P_{-}^{\prime}$, the following cases can occur:

1. $Z=P_{-}^{\prime}$ (equivalently $\left.\tilde{Z}=P^{\prime}\right)$. In this case $\delta\left([Z]^{B M}\right)=-[C] \otimes w_{1}$.

2. $Z \neq P_{-}^{\prime}$ (equivalently $\tilde{Z} \neq P^{\prime}$ ). In this, denoting by $\operatorname{deg}\left(Z \cap P_{x}^{\prime}\right)$ the degree of $Z \cap P_{x}^{\prime}$ in the projective space $P_{x}^{\prime}$ for generic $x \in B_{-}$, we'll have

$$
\delta\left([Z]^{B M}\right)=\operatorname{deg}\left(Z \cap P_{x}^{\prime}\right)[C] \otimes w_{r^{\prime}-1} .
$$

Taking into account the position of the divisor $c(\tilde{Z})$ with respect to $B$ (in $Q_{0}$ ), two subcases can occur:

(a) $c(\tilde{Z}) \cap B$ is 0 -dimensional. This implies that $\tilde{Z}$ does not intersect the generic fiber of $P^{\prime}$, hence $\operatorname{deg}\left(Z \cap P_{x}^{\prime}\right)=0$, and $\delta\left([Z]^{B M}\right)=0$.

(b) $c(\tilde{Z})$ contains $B$. This implies that $\tilde{Z}$ intersects all the fibers of the projective bundle $P^{\prime}$. Since we are in case 2 . the intersection with the generic fiber must be transversal, hence in this case we have $\operatorname{deg}\left(Z \cap P_{x}^{\prime}\right)>0$. Note that in this case $Z$ contains $P_{+}^{\prime \prime}=B_{+}$.

\section{Applications}

The following simple result shows how Theorem 3.9 is applied.

Proposition 4.1. Let $(X, g)$ be a Gauduchon surface with $b_{1}(X)=1, p_{g}(X)=$ 0 , $(E, h)$ a Hermitian rank 2-bundle on $X$ with $c_{1}(E) \notin 2 H^{2}(X, \mathbb{Z})$, and $\mathcal{D}$ a holomorphic structure on $\operatorname{det}(E)$. Suppose that

1. All reductions in $\mathcal{M}^{\mathrm{pst}}$ are regular,

2. The moduli space $\mathcal{M}^{\mathrm{pst}}$ associated with the data $(X, g, E, h, \mathcal{D})$ is compact.

For any Donaldson cohomology class $\nu \in H^{k-1}\left(\mathcal{B}^{*}, \mathbb{Q}\right)$ and any $\xi \in H_{k}^{B M}\left(\mathcal{M}^{\text {st }}, \mathbb{Q}\right)$ we have

$$
\sum_{\lambda \in \mathcal{D} e c(E)}\left\langle\nu_{\mathcal{P}_{\lambda}}, \delta_{\lambda} \xi\right\rangle=0
$$

where $\delta_{\lambda} \xi$ denotes the homological boundary of $\xi$ in $H_{k-1}\left(\mathcal{P}_{\lambda}, \mathbb{Q}\right)$. 
Proof. Denote by $\hat{\mathcal{M}}^{\text {pst }}$ the space obtained by blowing up all circles $C_{\lambda}$ in $\mathcal{M}^{\text {pst }}$. Using the Borel-Moore long exact sequence associated with the open embedding $\mathcal{M}^{\text {st }} \hookrightarrow \hat{\mathcal{M}}^{\text {pst }}\left[\overline{B H}\right.$, Section 1.6], and denoting by $j: \partial \hat{\mathcal{M}}^{\text {pst }} \hookrightarrow \hat{\mathcal{M}}^{\text {pst }}$ the inclusion map, we get $j_{*}(\delta \xi)=0$. Therefore

$$
\left\langle\left.\nu\right|_{\partial \hat{\mathcal{M}}^{\mathrm{pst}}}, \delta \xi\right\rangle=\left\langle\nu_{\hat{\mathcal{M}}^{\mathrm{pst}}}, j_{*}(\delta \xi)\right\rangle=0 .
$$

Here we used essentially that $\partial \hat{\mathcal{M}}^{\text {pst }}, \hat{\mathcal{M}}^{\text {pst }}$ are compact, hence their Borel-Moore homology coincides with their usual homology. It suffices to take into account that $\partial \hat{\mathcal{M}}^{\text {pst }}=\coprod_{\lambda \in \mathcal{D} e c(E)} \mathcal{P}_{\lambda}$.

We can now apply our results to the geometric problem formulated in the introduction concerning the Zariski closures of the extension families $\mathcal{P}_{\varepsilon}^{c}$. We are interested in the moduli space intervening in our program for proving the existence of curves on class VII surfaces. The following result will be used in [Te6]. We use the notations introduced in section 1

Proposition 4.2. Let $X$ be a minimal class VII surface with $H_{1}(X, \mathbb{Z}) \simeq \mathbb{Z}$ and $b_{2}(X)=3$, which is not an Enoki surface. Let $g$ be a Gauduchon metric on $X$ with $\operatorname{deg}_{g}(\mathcal{K})<0$. Then

1. The component $\mathcal{M}_{0} \in \pi_{0}\left(\mathcal{M}^{\mathrm{pst}}\right)$ of $\mathcal{P}_{\varepsilon}^{0}$ contains all four circles of reductions,

2. Let $i \in \mathfrak{I}$. The Zariski closure of $\mathcal{P}_{\varepsilon}^{e_{i}}$ in $\mathcal{M}^{\text {st }}$ has pure dimension 2, does not intersect $\mathcal{P}_{\varepsilon}^{0}$, and contains the curve $\mathcal{P}_{\varepsilon}^{e_{I}}$ for a subset $I \subset \mathfrak{I}$ of cardinal 2.

Proof. Put $\lambda_{0}:=\left\{0, e_{\mathfrak{I}}\right\}$, and, for $k \in \mathfrak{I}$, put $\lambda_{k}:=\left\{e_{k}, e_{\mathfrak{I} \backslash\{k\}}\right\}$. One has $r_{0}^{\prime}=3$, $r_{0}^{\prime \prime}=0, r_{e_{k}}^{\prime}=2, r_{e_{k}}^{\prime \prime}=1$.

1. We apply Proposition 4.1 to the analytic set $\mathcal{M}_{0}^{\text {st }}:=\mathcal{M}_{0} \cap \mathcal{M}^{\text {st }}$ taking $\nu:=$ $\mu(h) \cup \mu(u)$ for classes $h \in H_{1}(X, \mathbb{Z}), u \in H_{2}(X, \mathbb{Z})$. By Corollary 3.10 we have

$\delta\left[\Psi_{0}^{-1}\left(\mathcal{M}_{0}^{\mathrm{st}}\right)\right]=-\left[C_{0}\right] \otimes\left[\mathbb{P}\left(E_{0, y_{0}}^{\prime}\right)\right], \delta\left[\Psi_{e_{k}}^{-1}\left(\mathcal{M}_{0}^{\mathrm{st}}\right)\right]=a_{k}\left[C_{e_{k}}\right] \otimes\left[\mathbb{P}\left(E_{e_{k}, y_{k}}^{\prime} \oplus \bar{E}_{e_{k}, y_{k}}^{\prime \prime}\right)\right]$,

where $a_{k}:=\left\{\begin{array}{lll}1 & \text { if } \quad C_{\lambda_{k}} \subset \mathcal{M}_{0} \\ 0 & \text { if } \quad C_{\lambda_{k}} \not \subset \mathcal{M}_{0}\end{array}, y_{0} \in C_{0}\right.$ and $y_{k} \in C_{e_{k}}$. Using Theorem 2.2. and formula (4) we obtain

$$
\left\langle\gamma_{X}, h\right\rangle\left\{\left\langle e_{\mathfrak{J}}, u\right\rangle+\sum_{k} a_{k}\left\langle e_{k}-e_{\mathfrak{J} \backslash\{k\}}, u\right\rangle\right\}=0 \forall u \in H_{2}(X, \mathbb{Z}), \forall h \in H_{1}(X, \mathbb{Z}),
$$

Therefore $e_{\mathfrak{J}}+\sum_{k} a_{k}\left(e_{k}-e_{\mathfrak{J} \backslash\{k\}}\right)=0$, which holds only when $a_{k}=1$ for all $k \in \mathfrak{J}$. 2. Using the fact that $X$ is minimal one can prove as in Te5 that $\mathbb{P}_{y} \subset \mathcal{M}^{\text {st }}$ is a projective plane for any $y \in \operatorname{Pic}^{0}(X)_{(-\infty, \mathfrak{k})}$, the union

$$
\mathcal{P}_{\infty}^{0}:=\bigcup_{y \in \operatorname{Pic}^{0}(X)_{(-\infty, \mathfrak{e})}} \mathbb{P}_{y}
$$

is disjoint, and gives a Zariski open subset of $\mathcal{M}_{0}^{\text {st }}$ (see [Te6] for details). $\mathcal{P}_{\infty}^{0}$ is a $\mathbb{P}_{\mathbb{C}^{-}}^{2}$-bundle over the punctured disk $\operatorname{Pic}^{0}(X)_{(-\infty, \mathfrak{k})}$ via the natural projection. One can also check easily that $\mathcal{P}_{\varepsilon}^{e_{i}} \cap \mathcal{P}_{\infty}^{0}=\emptyset$. On the other hand, taking into account 1 ., we get $\mathcal{P}^{e_{i}} \subset \mathcal{M}_{0}^{\text {st }}$. This shows that $\mathcal{P}^{e_{i}}$ is contained in the complement $\mathcal{M}_{0}^{\text {st }} \backslash \mathcal{P}_{\infty}^{0}$, which is Zariski closed and nowhere dense. This proves that the Zariski closure $Z$ of $\mathcal{P}_{\varepsilon}^{e_{i}}$ in $\mathcal{M}^{\text {st }}$ has pure dimension 2 , and does not intersect $\mathcal{P}_{\varepsilon}^{0}$. For the last claim, let $\mathcal{I}_{k}$ be the set of irreducible components of $Z \cap \mathcal{O}_{\lambda_{k}}$ (as hypersurface of $\mathcal{O}_{\lambda_{k}}$ ). 
Taking $\nu:=\mu(h)$ in Proposition 4.1, and using the formulae explained in Example 3.2, we get

$$
0=\left\langle\gamma_{X}, h\right\rangle \sum_{k \in \mathfrak{I}} \sum_{Y \in \mathcal{I}_{k}} \operatorname{deg}\left(\Psi_{e_{k}}^{-1}(Y) \cap P_{e_{k}, z_{k}}^{\prime}\right),
$$

where $z_{k} \in B_{e_{k},-}$, and we agree to write $\operatorname{deg}\left(\Psi_{e_{k}}^{-1}(Y) \cap P_{e_{k}, z_{k}}^{\prime}\right)=-1$ if $\Psi_{e_{k}}^{-1}(Y)=$ $P_{e_{k},-}^{\prime}$. Supposing that $\mathcal{O}_{\lambda_{i}}$ is sufficiently small, we'll have $\mathcal{P}_{\varepsilon}^{e_{i}} \cap \mathcal{O}_{\lambda_{i}} \in \mathcal{I}_{i}$. This intersection corresponds to $P_{e_{i},-}^{\prime}$ via $\Psi_{e_{i}}$. Therefore at least a term on the right in (27) is -1 , hence there exists $k \in \mathfrak{J}$ and $Y \in \mathcal{I}_{k}$ such that $\left.\operatorname{deg}\left(\Psi_{e_{k}}^{-1}(Y) \cap P_{e_{k}, x}^{\prime}\right)\right)>0$. As explained in Example 3.2, this implies $P_{e_{k},+}^{\prime \prime} \subset \Psi_{e_{k}}^{-1}(Y)$, i.e., $\mathcal{P}_{\varepsilon}^{e_{\mathcal{I}} \backslash\{k\}} \subset Y$.

\section{REFERENCES}

[AK] G. Arone, M. Kankaanrinta: On the functoriality of the blow-up construction, Bull. Belg. Math. Soc. Simon Stevin Volume 17, Number 5, 821-832 (2010).

[BHPV] W. Barth, K. Hulek, Ch. Peters, A. Van de Ven: Compact complex surfaces, Springer (2004).

[BH] A. Borel, A. Haefliger: La classe d'homologie fondamentale d'un espace analytique, Bulletin de la SMF, 89, 461-513 (1961).

[Br] G. Bredon : Sheaf Theory, second edition, Graduate Texts in Mathematics, Springer (1997).

[Bu] N. Buchdahl: Hermitian-Einstein connections and stable vector bundles over compact complex surfaces, Math. Ann. 280, 625-648 (1988).

[BT] R. Bott, L. W. Tu: Differential forms in algebraic topology, Graduate Texts in Mathematics, Vol. 82, Springer (1982).

[Do] A. Dold: Lectures on Algebraic Topology, Springer (1980).

[DK] S. Donaldson, P. Kronheimer: The Geometry of Four-Manifolds, Oxford Univ. Press (1990).

[Gau] P. Gauduchon: Sur la 1-forme de torsion d'une variété hermitienne compacte, Math. Ann. 267, 495-518 (1984).

[GR] H. Grauert, R. Remmert: Extension of Analytic Objects, in Several Complex Variables VII, Encyclopaedia of Mathematical Sciences, Vol. 74, eds: H. Grauert, Th. Peternell, R. Remmert, Springer (1994).

[LT] M. Lübke, A. Teleman: The Kobayashi-Hitchin correspondence, World Scientific Publishing Co. (1995).

[Na] I. Nakamura: Towards classification of non-Kählerian surfaces, Sugaku Expositions vol. 2, No 2 , 209-229 (1989).

[Sp] E. Spanier: Algebraic topology, McGraw-Hill (1966).

[Te1] A. Teleman: Donaldson theory on non-Kählerian surfaces and class VII surfaces with $b_{2}=1$, Invent. math. 162, 493-521 (2005).

[Te2] A. Teleman: Harmonic sections in sphere bundles, normal neighborhoods of reduction loci, and instanton moduli spaces on definite 4-manifolds, Geometry \& Topology 11, 1681-1730, (2007).

[Te3] A. Teleman: Instantons and holomorphic curves on class VII surfaces, Annals of Mathematics 172, 1749-1804 (2010).

[Te4] A. Teleman: A variation formula for the determinant line bundle. Compact subspaces of moduli spaces of stable bundles over class VII surfaces, arXiv:1309.0350 [math.CV].

[Te5] A. Teleman: Instanton moduli spaces on non-Kählerian surfaces. Holomorphic models around the reduction loci, arXiv:1411.4985 [math.DG].

[Te6] A. Teleman: Class VII surfaces with $b_{2}=3$, in preparation.

Aix Marseille Université, CNRS, Centrale Marseille, I2M, UMR 7373, 13453 MarSEILle, France 